\title{
Weak deflection gravitational lensing for photons coupled to Weyl tensor in a Schwarzschild black hole
}

\author{
Wei-Guang Cao ${ }^{1,2}$, Yi Xie ${ }^{1,2, a}$ \\ ${ }^{1}$ School of Astronomy and Space Science, Nanjing University, Nanjing 210093, China \\ ${ }^{2}$ Key Laboratory of Modern Astronomy and Astrophysics, Nanjing University, Ministry of Education, Nanjing 210093, China
}

Received: 12 February 2018 / Accepted: 27 February 2018 / Published online: 7 March 2018

(C) The Author(s) 2018. This article is an open access publication

\begin{abstract}
Beyond the Einstein-Maxwell model, electromagnetic field might couple with gravitational field through the Weyl tensor. In order to provide one of the missing puzzles of the whole physical picture, we investigate weak deflection lensing for photons coupled to the Weyl tensor in a Schwarzschild black hole under a unified framework that is valid for its two possible polarizations. We obtain its coordinate-independent expressions for all observables of the geometric optics lensing up to the second order in the terms of $\varepsilon$ which is the ratio of the angular gravitational radius to angular Einstein radius of the lens. These observables include bending angle, image position, magnification, centroid and time delay. The contributions of such a coupling on some astrophysical scenarios are also studied. We find that, in the cases of weak deflection lensing on a star orbiting the Galactic Center Sgr A*, Galactic microlensing on a star in the bulge and astrometric microlensing by a nearby object, these effects are beyond the current limits of technology. However, measuring the variation of the total flux of two weak deflection lensing images caused by the Sgr A* might be a promising way for testing such a coupling in the future.
\end{abstract}

\section{Introduction}

Gravitational lensing has become an invaluable tool in astronomy, cosmology and gravitational physics [1-4]. The intrinsic essence of gravitational lensing is the interaction between electromagnetic and gravitational fields. Beyond the standard Einstein-Maxwell theory, the effect of one-loop vacuum polarization on photons was considered under different spacetimes and found to be extremely small [5-15]. Extended models of the coupling were also investigated for various physical circumstances [16-30].

\footnotetext{
a e-mail: yixie@nju.edu.cn
}

The Weyl tenser can also play as a mediator to couple the electromagnetic and gravitational fields. Such a coupling has been widely investigated in several contexts [31-43]. Recently, strong deflection gravitational lensing for photons coupled to the Weyl tensor has received much attention due to the deployment of direct observation on the supermassive black hole at the Galactic center, Sgr A*, by the Event Horizon Telescope. ${ }^{1}$ The unique feature of the strong deflection lensing is the relativistic images of photons winding several loops around the lens $[44,45]$, which can not be generated in a weak gravitational field. Focusing on photons coupled to the Weyl tensor in a Schwarzschild black hole, the strong deflection lensing was studied $[46,47]$. It was then extended to a more complicated background by considering a Kerr black hole [48].

However, the relativistic images of Sgr A* are extremely faint [49-51] and, therefore, exceedingly difficult to detect. As an alternative, the primary and secondary images of weak deflection gravitational lensings are much easier to observe and they have been extensively used in astronomy and cosmology [1-3]. Weak deflection lensings can also provide insights on modified theories of gravity [52-54] and clues on the interaction between electromagnetic and gravitational fields. In this work, we will study weak deflection lensing for photons coupled to Weyl tensor in a Schwarzschild black hole, which was absent in the literature. By focusing on coordinate-invariant quantities, we obtain all of its geometric optics lensing observables, which include bending angle, image position, magnification, centroid and time delay. These observables are worked out to the second order in the perturbation parameter $\varepsilon$ which is the ratio of the angular gravitational radius to angular Einstein radius of the lens. The results are represented in a unified form which is valid for both of two polarization directions of the Weyl coupling.

\footnotetext{
$\overline{1 \text { http://www.eventhorizontelescope.org/. }}$
} 
In Sect. 2, after the unified effective metric for photons coupled to the Weyl tensor in a Schwarzschild black hole with two polarization directions is briefly reviewed, we will derive its light bending angle that is expressed with invariant quantities. The lensing observables, including positions, magnifications and time delay of images, are obtained in Sect. 3 and relations between them are represents in Sect. 4. We work out practical observables of the lensing and investigate its observability for several astrophysical scenarios in Sect. 5. Finally, in Sect. 6, we summarize and discuss our results.

\section{Effective metric and light bending}

\subsection{Effective metric}

We consider a Schwarzschild black hole with mass $M_{\bullet}$ as the lens, and set the observer and the source in the asymptotically flat region of its spacetime. We assume that it is vacuum outside the lens. When a photon couples to the Weyl tensor in the background of the Schwarzschild black hole, its worldline will no longer follow the null geodesic. However, it was found $[39,46]$ that the geodesic rule can be recovered by taking an effective metric for such a coupling. This metric can be written as

$\mathrm{d} s^{2}=-A(r) \mathrm{d} t^{2}+B(r) \mathrm{d} r^{2}+C(r) \mathrm{d} \Omega^{2}$,

where $r$ is the radial coordinate and $\mathrm{d} \Omega^{2}=\mathrm{d} \theta^{2}+\sin ^{2} \theta \mathrm{d} \varphi^{2}$. The functions $A(r), B(r)$ and $C(r)$ are

$A(r)=B(r)^{-1}=1-\frac{2 m \bullet}{r}$

and

$C(r)=\left(\frac{r^{3}+16 \alpha m_{\bullet}}{r^{3}-8 \alpha m_{\bullet}}\right)^{s} r^{2}$,

where $\alpha$ is a constant with dimension of [Length $]^{2}$ characterising strength of the coupling between the photon and the Weyl tensor, $m_{\bullet}=G M_{\bullet} / c^{2}$ is the gravitational radius of the Schwarzschild black hole, and $s$ is an constant. We use $s$ to unify the expression of $C(r)$ for two different polarizations of the photon respectively along $l_{\mu}(\mathrm{PPL})$ and $m_{\mu}$ (PPM) (see $[39,46]$ for more details):

$s= \begin{cases}+1 & \text { for PPL, } \\ -1 & \text { for PPM. }\end{cases}$

In fact, the results of weak deflection lensing that we obtain in the following parts are also valid when $s$ takes other real numbers with different physical interpretations.

Before we perform detailedly and lengthy calculation on the light bending and its resulting lensing observables, it is worth mentioning that the parameterized second-order postNewtonian formalism for weak deflection lensing established in Refs. [52,53], which is called "Keeton-Petters formalism" for short hereafter, cannot be applied to the spacetime (1) in this paper. Keeton-Petters formalism is valid for a static, spherically symmetric and asymptotically flat spacetime, whose metric is written in the standard Schwarzschild coordinates denoted by overbar with coefficients: [52,53]

$A_{\mathrm{KP}}(\bar{r})=1+2 a_{1} \frac{\phi}{c^{2}}+2 a_{2}\left(\frac{\phi}{c^{2}}\right)^{2}+2 a_{3}\left(\frac{\phi}{c^{2}}\right)^{3}+\cdots$,
$B_{\mathrm{KP}}(\bar{r})=1-2 b_{1} \frac{\phi}{c^{2}}+4 b_{2}\left(\frac{\phi}{c^{2}}\right)^{2}-8 b_{3}\left(\frac{\phi}{c^{2}}\right)^{3}+\cdots$,
$C_{\mathrm{KP}}(\bar{r})=\bar{r}^{2}$.

Here, $\phi$ is the Newtonian potential with

$\frac{\phi}{c^{2}}=-\frac{m_{\bullet}}{\bar{r}}$,

and $a_{1,2,3}$ and $b_{1,2,3}$ are dimensionless and numerical parameters. However, after transforming (1) from its current coordinates to the standard Schwarzschild ones, we find that $b_{1,2,3}$ depend on the radial coordinate rather than numerical ones, such as

$b_{1}(\bar{r})=1+24 s \frac{\alpha}{\bar{r}^{2}}$.

The additional $\bar{r}$-dependent terms have to be taken into account when evaluating the integral of the light bending angle (see next subsection for details); however, they are absent in the Keeton-Petters formalism. This issue was also recognized for Solar System tests of a scalar-tensor gravity [55]. One exception is the trivial case of $\alpha=0$ in which the metric (1) reduce to the Schwarzschild black hole in the standard coordinates. Therefore, due to the fact that such a formalism cannot be directly employed, we stick to the metric (1) and perform all of the indispensable calculation for proceeding our investigation.

\subsection{Light bending}

For a light ray propagating through spacetime (1), the distance of closet approach $r_{0}$ and the impact parameter $b$ of the light ray satisfy the relation as [56]

$C\left(r_{0}\right)=b^{2} A\left(r_{0}\right)$.

In the scenario of weak deflection lensing, $r_{0}$ and $b$ are much larger than $m$ • which leads to the Taylor expanded solution to $(10)$ as

$\frac{r_{0}}{b}=1-\sum_{n=1}^{6} \mathfrak{b}_{n} q^{n}+\mathscr{O}\left(q^{7}\right)$

with small parameter

$q \equiv \frac{m_{\bullet}}{b}$. 
The first two coefficients $\mathfrak{b}_{1}$ and $\mathfrak{b}_{2}$ are

$\mathfrak{b}_{1}=1+12 s \bar{\alpha}$,

$\mathfrak{b}_{2}=\frac{3}{2}+36 s \bar{\alpha}+24 s \bar{\alpha}^{2}(15 s-2)$,

where we define a dimensionless parameter as

$\bar{\alpha} \equiv \frac{\alpha}{b^{2}} ;$

the details of other $\mathfrak{b}_{n}(n=3, \ldots, 6)$ can be found in Appendix A. When the coupling vanishes, i.e., $\bar{\alpha}=0$, Eq. (11) returns to the one for the Schwarzschild black hole in Einstein's general relativity (GR) [52].

Following the standard procedure, e.g. [56], the bending angle can be obtained as $[49,56]$

$\hat{\alpha}\left(r_{0}\right)=\int_{r_{0}}^{\infty} \frac{2 \sqrt{B(r)}}{\sqrt{C(r)} \sqrt{\frac{C(r)}{C\left(r_{0}\right)} \frac{A\left(r_{0}\right)}{A(r)}-1}} \mathrm{~d} r-\pi$,

which can be written in the form of a series for weak deflection lensing as [52]

$\hat{\alpha}(h)=\sum_{n=1}^{6} \mathfrak{a}_{n} h^{n}+\mathscr{O}\left(h^{7}\right)$

with small parameter

$h \equiv \frac{m_{\bullet}}{r_{0}}$.

The coefficients $\mathfrak{a}_{n}$ for $n=1$ and 2 are

$$
\begin{aligned}
\mathfrak{a}_{1}= & 4+32 s \bar{\alpha}_{0}, \\
\mathfrak{a}_{2}= & \frac{15}{4} \pi-4+s \bar{\alpha}_{0}\left(\frac{135}{2} \pi-144\right) \\
& +s \bar{\alpha}_{0}^{2}\left[\left(\frac{1125}{2} \pi-1152\right) s-75 \pi\right],
\end{aligned}
$$

where the dimensionless parameter $\bar{\alpha}_{0}$ is defined as

$\bar{\alpha}_{0} \equiv \frac{\alpha}{r_{0}^{2}}$.

For testing photons coupled to the Weyl tensor in the Solar System, the leading term of $\hat{\alpha}(h) \approx \mathfrak{a}_{1} h$ was calculated previously [57]; and our result is in agreement with that. Other higher-order coefficients $\mathfrak{a}_{n}(n=3, \ldots, 6)$ can be found in Appendix A. The deflection angle (17) can go back to the one for the Schwarzschild black hole in GR [52] when $\bar{\alpha}=0$. However, such an expression depends on coordinate of $r_{0}$ and should be transformed into an gauge-invariant form. With the help of Eq. (11), we can replace the distance of closet approach $r_{0}$ with the impact parameter $b$ and obtain

$\hat{\alpha}(b)=\sum_{n=1}^{6} \hat{\alpha}_{n} q^{n}+\mathscr{O}\left(q^{7}\right)$, where

$\hat{\alpha}_{1}=4+32 s \bar{\alpha}$,

$\hat{\alpha}_{2}=\pi\left[\frac{15}{4}+\frac{135}{2} s \bar{\alpha}+\frac{75}{2} s \bar{\alpha}^{2}(15 s-2)\right]$,

and higher-order coefficients $\hat{\alpha}_{n}(n=3, \ldots, 6)$ can be found in Appendix A. It can be easily checked that the gaugeinvariant deflection angle (22) can return to the one for the Schwarzschild black hole in GR [52] when the coupling vanishes.

\section{Image positions, magnifications and time delay}

After the deflection angle has been obtained, we can determine the image positions, magnifications, and time delay of the weak deflection lensing for photons coupled to the Weyl tensor in the Schwarzschild black hole. Denoting $d_{\mathrm{L}}, d_{\mathrm{S}}$ and $d_{\mathrm{LS}}$ as angular diameter distances between the observer, lens and the source, we adopt the general lens equation as $[49,50]$

$\tan \mathscr{B} \equiv \tan \vartheta-D[\tan \vartheta+\tan (\hat{\alpha}-\vartheta)]$,

where $\mathscr{B}$ is the angular position of the source, $\vartheta=$ $\arcsin \left(b / d_{\mathrm{L}}\right)$ is the angular position of the image and $D=$ $d_{\mathrm{LS}} / d_{\mathrm{S}}$. Following the convention of Refs. [52-54], angles of image positions are set to be positive so that the angular position of the source $\mathscr{B}$ is positive if the image is on the same side of the lens as the source while $\mathscr{B}$ is negative if the image is on the opposite side. We also define scaled variables [52-54]

$\beta=\frac{\mathscr{B}}{\vartheta_{\mathrm{E}}}, \quad \theta=\frac{\vartheta}{\vartheta_{\mathrm{E}}}, \quad \hat{\tau}=\frac{\tau}{\tau_{\mathrm{E}}}, \quad \varepsilon=\frac{\vartheta_{\bullet}}{\vartheta_{\mathrm{E}}}$,

where $\vartheta_{\bullet}=\arctan \left(m_{\bullet} / d_{\mathrm{L}}\right)$ is the angular gravitational radius at distance $d_{\mathrm{L}}, \tau$ is the time delay between images, the angular Einstein ring radius is

$\vartheta_{\mathrm{E}}=\sqrt{\frac{4 m_{\bullet} d_{\mathrm{LS}}}{d_{\mathrm{L}} d_{\mathrm{S}}}}$

and the time scale is

$\tau_{\mathrm{E}}=4 \frac{m \bullet}{c}$.

Then, it is assumed that solution to the lens equation (25) can be expressed in the form of a series as

$\theta=\theta_{0}+\varepsilon \theta_{1}+\varepsilon^{2} \theta_{2}+\mathscr{O}\left(\varepsilon^{3}\right)$,

where $\theta_{0}, \theta_{1}$ and $\theta_{2}$ are respectively the zeroth-order, firstorder and second-order terms for the image position in the weak deflection lensing. With that, the bending angle (22) can be written as 


$$
\begin{aligned}
\hat{\alpha}= & 4 \frac{\varepsilon}{\theta_{0}}(1+8 s \bar{\alpha})+\frac{\varepsilon^{2}}{\theta_{0}^{2}}\left[\frac{15}{4} \pi-4 \theta_{1}+\frac{1}{2} s \bar{\alpha}(135 \pi\right. \\
& \left.\left.-64 \theta_{1}\right)+\frac{75}{2} \pi s \bar{\alpha}^{2}(15 s-2)\right]+\frac{\varepsilon^{3}}{\theta_{0}^{3}}\left\{\frac{128}{3}\right. \\
& -\frac{15}{2} \pi \theta_{1}-4 \theta_{0} \theta_{2}+4 \theta_{1}^{2}+\frac{32}{3} D^{2} \theta_{0}^{4}+s \bar{\alpha}\left(\frac{6144}{5}\right. \\
& \left.-135 \pi \theta_{1}+32 \theta_{1}^{2}-32 \theta_{0} \theta_{2}+\frac{256}{3} D^{2} \theta_{0}^{4}\right) \\
& -s \bar{\alpha}^{2}\left[\left(1125 \pi \theta_{1}-\frac{663552}{35}\right) s-150 \pi \theta_{1}+\frac{73728}{35}\right] \\
& \left.+\frac{524288}{105} s \bar{\alpha}^{3}\left(24 s^{2}-6 s+1\right)\right\}+\mathscr{O}\left(\varepsilon^{4}\right),
\end{aligned}
$$

and the lens equation (25) can be found as

$$
\begin{aligned}
0= & 4 D \frac{\varepsilon}{\theta_{0}}\left(\beta \theta_{0}-\theta_{0}^{2}+1+8 s \bar{\alpha}\right)+\frac{\varepsilon^{2}}{\theta_{0}^{2}} D\left[\frac{15}{4} \pi\right. \\
& -4 \theta_{1}\left(\theta_{0}^{2}+1\right)+\frac{1}{2} s \bar{\alpha}\left(135 \pi-64 \theta_{1}\right) \\
& \left.+\frac{75}{2} \pi s \bar{\alpha}^{2}(15 s-2)\right]+\frac{\varepsilon^{3}}{\theta_{0}^{3}} D\left\{64-\frac{15}{2} \pi \theta_{1}\right. \\
& -64 D \theta_{0}^{2}-4 \theta_{0} \theta_{2}+4 \theta_{1}^{2}+\frac{64}{3} \beta^{3} D^{2} \theta_{0}^{3}+\frac{224}{3} D^{2} \theta_{0}^{4} \\
& -4 \theta_{0}^{3} \theta_{2}-\frac{64}{3} D^{2} \theta_{0}^{6}+s \bar{\alpha}\left(\frac{8704}{5}-135 \pi \theta_{1}\right. \\
& \left.-1024 D \theta_{0}^{2}-32 \theta_{0} \theta_{2}+32 \theta_{1}^{2}+\frac{1792}{3} D^{2} \theta_{0}^{4}\right) \\
& +s \bar{\alpha}^{2}\left[s\left(\frac{806912}{35}-1125 \pi \theta_{1}-4096 D \theta_{0}^{2}\right)\right. \\
& \left.\left.+150 \pi \theta_{1}-\frac{73728}{35}\right]+\frac{32768}{105} s \bar{\alpha}^{3}\left(419 s^{2}-96 s+16\right)\right\} \\
& +\mathscr{O}\left(\varepsilon^{4}\right),
\end{aligned}
$$

which will be used to work out the observables.

\subsection{Image positions}

By making the coefficients of $\epsilon, \epsilon^{2}$ and $\epsilon^{3}$ in (31) vanish, we can find out $\theta_{n}(n=0,1,2)$. The first term gives that

$\beta=\theta_{0}-\frac{1+8 s \bar{\alpha}}{\theta_{0}}$,

which leads to the zeroth-order image position for the weak deflection lensing as

$\theta_{0}=\frac{1}{2}(\beta+\eta)$

where

$\eta=\sqrt{\beta^{2}+4+32 s \bar{\alpha}}$.

It is worth mentioning that the negative solution of $\theta_{0}$ is neglected due to our convention that the angular position of an image is set to be positive. The positive- and negativeparity images can respectively be found by using $\beta>0$ and $\beta<0$ (see next section for details). If $s \bar{\alpha}>0$, it will make $\theta_{0}$ bigger than its corresponding values for the Schwarzschild black hole in the absence of such a coupling to the Weyl tensor; if $s \bar{\alpha}<0, \theta_{0}$ will become smaller. Additionally, Eq. (33) itself also imposes a bound on $\bar{\alpha}$. In order to ensure that $\eta$ and resulting $\theta_{0}$ are real, it demands that

$s \bar{\alpha} \geq-\frac{1}{8}-\frac{\beta^{2}}{32}$.

For the special case of $\beta=0$ and $s=-1$, it implies $\bar{\alpha} \leq 1 / 8$ so that $\alpha \lesssim 6 \times 10^{16} \mathrm{~m}^{2}$ where $b$ is assumed to be the radius of the Sun by considering the Sun as the lens. Such a specific bound is consistent with but looser than the one obtained by the Solar System test on the deflection of light due to the Sun [57].

The coefficient of $\epsilon^{2}$ in (31) does not depend on $\beta$ explicitly so that, after substituting (33), we can obtain the firstorder correction to the image position as

$\theta_{1}=\frac{15}{16} \pi \frac{1+18 s \bar{\alpha}+10 s(15 s-2) \bar{\alpha}^{2}}{\theta_{0}^{2}+1+8 s \bar{\alpha}}$.

It can be easily checked that when $\bar{\alpha}=0, \theta_{1}$ will return to its familiar value for the Schwarzschild black hole in GR [52].

Vanishing the coefficient of $\epsilon^{3}$ in (31) yields the secondorder term as

$\theta_{2}=\mathfrak{p} \sum_{n=0}^{5} \mathfrak{p}_{n} \bar{\alpha}^{n}$

where the factor $\mathfrak{p}$ and first two coefficients $\mathfrak{p}_{0}$ and $\mathfrak{p}_{1}$ are

$$
\begin{aligned}
\mathfrak{p}= & \frac{1}{\theta_{0}\left(\theta_{0}^{2}+1+8 s \bar{\alpha}\right)^{3}}, \\
\mathfrak{p}_{0}= & \frac{8}{3} D^{2} \theta_{0}^{8}+D\left(\frac{64}{3} D-16\right) \theta_{0}^{6} \\
& +\left(\frac{88}{3} D^{2}-32 D+16\right) \theta_{0}^{4} \\
& +\theta_{0}^{2}\left(\frac{16}{3} D^{2}-16 D+32-\frac{225}{128} \pi^{2}\right) \\
& -\frac{16}{3} D^{2}+16-\frac{225}{256} \pi^{2}, \\
\mathfrak{p}_{1}= & s \frac{64}{3} D^{2} \theta_{0}^{8}+D\left(\frac{1024}{3} D-256\right) \theta_{0}^{6} \\
& +\left(704 D^{2}-768 D+\frac{2176}{5}\right) \theta_{0}^{4} \\
& +\left(\frac{512}{3} D^{2}-512 D+\frac{5632}{5}-\frac{2025}{32} \pi^{2}\right) \theta_{0}^{2} \\
& \left.-\frac{640}{3} D^{2}+\frac{3456}{5}-\frac{2475}{64} \pi^{2}\right] .
\end{aligned}
$$


The higher-order coefficients $\mathfrak{p}_{n}(n=2, \ldots, 5)$ can be found in Appendix B. If the coupling to the Weyl tensor is absent, $\theta_{2}$ will have its value as the same as the one for the Schwarzschild black hole [52].

\subsection{Magnifications}

At angular position $\vartheta$, the signed magnification $\mu$ is [58]

$\mu(\vartheta)=\left[\frac{\sin \mathscr{B}(\vartheta)}{\sin \vartheta} \frac{\mathrm{d} \mathscr{B}(\vartheta)}{\mathrm{d} \vartheta}\right]^{-1}$.

With scaled variables (26), we can have a series of $\mu$ expanded in terms of $\epsilon$ as

$\mu=\mu_{0}+\varepsilon \mu_{1}+\varepsilon^{2} \mu_{2}+\mathscr{O}\left(\varepsilon^{3}\right)$,

where the zeroth-order, first-order and second-order terms are

$\mu_{0}=\frac{\theta_{0}^{4}}{\theta_{0}^{4}-(1+8 s \bar{\alpha})^{2}}$,

$\mu_{1}=-\frac{15}{16} \pi \theta_{0}^{3} \frac{1+18 s \bar{\alpha}+10 s(15 s-2) \bar{\alpha}^{2}}{\left(\theta_{0}^{2}+1+8 s \bar{\alpha}\right)^{3}}$,

$\mu_{2}=\mathfrak{m} \sum_{n=0}^{6} \mathfrak{m}_{n} \bar{\alpha}^{n}$

In the expression of $\mu_{2}$, the factor $\mathfrak{m}$ and the first two coefficients $\mathfrak{m}_{0}$ and $\mathfrak{m}_{1}$ are

$$
\begin{aligned}
\mathfrak{m}= & \frac{\theta_{0}^{2}}{\left(\theta_{0}^{2}+1+8 s \bar{\alpha}\right)^{5}\left(\theta_{0}^{2}-1-8 s \bar{\alpha}\right)}, \\
\mathfrak{m}_{0}= & \frac{8}{3} D^{2} \theta_{0}^{8}+\left(48 D^{2}-32 D-32\right) \theta_{0}^{6} \\
& +\left(\frac{272}{3} D^{2}-64 D+\frac{675}{128} \pi^{2}-64\right) \theta_{0}^{4} \\
& +\left(48 D^{2}-32 D-32\right) \theta_{0}^{2}+\frac{8}{3} D^{2}, \\
\mathfrak{m}_{1}= & s\left[\frac{128}{3} D^{2} \theta_{0}^{8}+\left(1152 D^{2}-768 D-\frac{4352}{5}\right) \theta_{0}^{6}\right. \\
& +\left(\frac{8704}{3} D^{2}-2048 D+\frac{6075}{32} \pi^{2}-\frac{11264}{5}\right) \theta_{0}^{4} \\
& \left.+\left(1920 D^{2}-1280 D-\frac{6912}{5}\right) \theta_{0}^{2}+128 D^{2}\right],
\end{aligned}
$$

and the higher-order coefficients $\mathfrak{m}_{n}(n=2, \ldots 6)$ can be found in Appendix B. It can be checked that $\mu$ will be divergent if $s \bar{\alpha}$ equals to either $-\left(\theta_{0}^{2}+1\right) / 8$ or $\left(\theta_{0}^{2}-1\right) / 8$. When $\bar{\alpha}=0, \mu_{n}(n=0,1,2)$ can also return to their familiar values for the Schwarzschild black hole [52].

\subsection{Time delay}

The time delay is the difference between the light travel time with and without the lens and it can be expressed as

$c \tau=T\left(R_{\mathrm{src}}\right)+T\left(R_{\mathrm{obs}}\right)-\frac{d_{\mathrm{S}}}{\cos \mathscr{B}}$

where $R_{\mathrm{src}}$ and $R_{\mathrm{obs}}$ are the radial coordinates of the source and observer and they have the relations as [52]

$$
\begin{aligned}
R_{\text {src }} & =\left(d_{\mathrm{LS}}^{2}+d_{\mathrm{S}}^{2} \tan ^{2} \mathscr{B}\right)^{1 / 2}, \\
R_{\text {obs }} & =d_{\mathrm{L}} .
\end{aligned}
$$

The function $T(R)$ has the form as

$T(R)=\int_{r_{0}}^{R}\left|\frac{\mathrm{d} t}{\mathrm{~d} r}\right| \mathrm{d} r$

and it can be integrated and expanded as

$T(R)=T_{0}+r_{0} \sum_{n=1}^{3} T_{n} h^{n}+\mathscr{O}\left(h^{4}\right)$,

where the first two terms are

$$
\begin{aligned}
T_{0}= & \sqrt{R^{2}-r_{0}^{2}}, \\
T_{1}= & \frac{\sqrt{1-\xi^{2}}}{1+\xi}+2 \ln \left(\frac{1+\sqrt{1-\xi^{2}}}{\xi}\right) \\
& +12 s \bar{\alpha}_{0} \frac{\sqrt{1-\xi^{2}}}{1+\xi}(\xi+2)
\end{aligned}
$$

with

$\xi=\frac{r_{0}}{R}$

and higher-order terms $T_{3}$ and $T_{4}$ can be found in Appendix B. It is obvious that $T_{0}$ is not affected by the coupling to the Weyl tensor, but $T_{1}$ has the Shapiro delay term with an additional correction proportional to $s \bar{\alpha}_{0}$ which is consistent with the result obtained for the Solar System test [57]. When $\bar{\alpha}_{0}=0, T_{n}(n=1,2,3)$ can return to their values for the Schwarzschild black hole [52].

After replacing $r_{0}$ with $b$ by using Eq. (11) and substituting (50) and (51), we can have the scaled time delay [see Eq. (26)] in a series as

$\hat{\tau}=\hat{\tau}_{0}+\varepsilon \hat{\tau}_{1}+\mathscr{O}\left(\varepsilon^{2}\right)$,

where

$$
\begin{aligned}
\hat{\tau}_{0}= & \frac{1}{2}\left[1+\beta^{2}-\theta_{0}^{2}-\ln \left(\frac{d_{\mathrm{L}} \theta_{0}^{2} \vartheta_{\mathrm{E}}^{2}}{4 d_{\mathrm{LS}}}\right)+24 s \bar{\alpha}\right], \\
\hat{\tau}_{1}= & \frac{15 \pi}{8 \theta_{0}}\left\{\frac{1}{2}+\frac{s \bar{\alpha}}{\theta_{0}^{2}+1+8 s \bar{\alpha}}\left[7+3 \theta_{0}^{2}+\bar{\alpha}\left(15 \theta_{0}^{2} s\right.\right.\right. \\
& \left.\left.\left.+111 s-2 \theta_{0}^{2}-2\right)+s \bar{\alpha}^{2}(720 s-96)\right]\right\} .
\end{aligned}
$$


Although it is also possible to obtain the $\mathscr{O}\left(\varepsilon^{2}\right)$ term for $\hat{\tau}$, that is less vital than the $\mathscr{O}\left(\varepsilon^{2}\right)$ corrections to $\theta$ and $\mu$. If $s \bar{\alpha}>0$, it will make $\hat{\tau}_{0}$ bigger than its corresponding value in the absence of the coupling to the Weyl tensor; if $s \bar{\alpha}<0$, $\hat{\tau}_{0}$ will become smaller. When such a coupling vanishes, $\hat{\tau}_{0}$ and $\hat{\tau}_{1}$ can go back to their values in GR [52].

\section{Relations between lensing observables}

Considering photons coupled to the Weyl tensor in the Schwarzschild black hole, we can find some relations between lensing observables given in the previous section.

\subsection{Position relations}

With Eq. (33), we can respectively obtain the positive- and negative-parity images at the leading order by specifying $\beta>$ 0 and $\beta<0$ as

$\theta_{0}^{ \pm}=\frac{1}{2}(\eta \pm|\beta|)$

which also leads to

$\theta_{0}^{+}-\theta_{0}^{-}=|\beta|$,

and

$\theta_{0}^{+} \theta_{0}^{-}=1+8 s \bar{\alpha}$.

It is clear that the value of $\theta_{0}^{+}-\theta_{0}^{-}$is not affected by the coupling to the Weyl tensor and $\theta_{0}^{+} \theta_{0}^{-}$is dependent on the coupling only. When such a coupling vanishes, $\theta_{0}^{ \pm}$and $\theta_{0}^{+} \theta_{0}^{-}$ will have their values in GR [52]. If $s \bar{\alpha}>0$, then it will make $\theta_{0}^{ \pm}$and $\theta_{0}^{+} \theta_{0}^{-}$bigger than their corresponding values in the absence of such a coupling to the Weyl tensor; and vice versa.

According to Eqs. (36) and (60), we can have the firstorder corrections to the image positions as

$\theta_{1}^{ \pm}=\frac{15}{8} \pi \frac{1+18 s \bar{\alpha}+10 s \bar{\alpha}^{2}(15 s-2)}{\eta(\eta \pm|\beta|)}$.

They generate two relations that one is

$\theta_{1}^{+}+\theta_{1}^{-}=\frac{15}{16} \pi \frac{1+18 s \bar{\alpha}+10 s \bar{\alpha}^{2}(15 s-2)}{1+8 s \bar{\alpha}}$,

which is independent on the angular position of source; and the other is

$\theta_{1}^{+}-\theta_{1}^{-}=-\frac{15}{16} \pi|\beta| \frac{1+18 s \bar{\alpha}+10 s \bar{\alpha}^{2}(15 s-2)}{\eta(1+8 s \bar{\alpha})}$.

which is source-dependent.

Based (37), we can obtain the second-order corrections to the image positions as

$\theta_{2}^{ \pm}=\mathfrak{P}_{ \pm}\left(\sum_{n=0}^{5} \mathfrak{P}_{n} \bar{\alpha}^{n} \pm \eta|\beta| \sum_{n=0}^{4} \mathfrak{P}_{n}^{\prime} \bar{\alpha}^{n}\right)$, where the factors $\mathfrak{P}_{ \pm}$and the first two coefficients of $\mathfrak{P}_{n}$ and $\mathfrak{P}_{n}^{\prime}(n=0,1)$ are

$$
\begin{aligned}
\mathfrak{P}_{ \pm}= & \frac{1}{\eta^{3}(\eta \pm|\beta|)^{4}}, \\
\mathfrak{P}_{0}= & \frac{64}{3} D^{2} \beta^{8}+D\left(\frac{1024}{3} D-128\right) \beta^{6} \\
& +\left(\frac{5056}{3} D^{2}-1024 D+128\right) \beta^{4} \\
& +\left(\frac{8576}{3} D^{2}-2304 D+768-\frac{225}{16} \pi^{2}\right) \beta^{2} \\
& +\frac{2560}{3} D^{2}-1024 D+1024-\frac{675}{16} \pi^{2},
\end{aligned}
$$

$$
\begin{aligned}
\mathfrak{P}_{1}= & s\left[\frac{512}{3} D^{2} \beta^{8}+D\left(\frac{16384}{3} D-2048\right) \beta^{6}\right. \\
& +\left(40448 D^{2}-24576 D+\frac{17408}{5}\right) \beta^{4} \\
& +\left(\frac{274432}{3} D^{2}-73728 D+\frac{135168}{5}-\frac{2025}{4} \pi^{2}\right) \beta^{2} \\
& \left.+\frac{102400}{3} D^{2}-40960 D+\frac{221184}{5}-\frac{7425}{4} \pi^{2}\right] \\
\mathfrak{P}_{0}^{\prime}= & \frac{64}{3} D^{2} \beta^{6}+D\left(\frac{896}{3} D-128\right) \beta^{4} \\
& +\left(\frac{3392}{3} D^{2}-768 D+128\right) \beta^{2} \\
& +\frac{3328}{3} D^{2}-1024 D+512-\frac{225}{16} \pi^{2} \\
\mathfrak{P}_{1}^{\prime}= & s \frac{512}{3} D^{2} \beta^{6}+D\left(\frac{14336}{3} D-2048\right) \beta^{4} \\
& +\left(27136 D^{2}-18432 D+\frac{17408}{5}\right) \beta^{2} \\
& \left.+\frac{106496}{3} D^{2}-32768 D+\frac{90112}{5}-\frac{2025}{4} \pi^{2}\right]
\end{aligned}
$$

and higher-order coefficients $\mathfrak{P}_{n}(n=2, \ldots, 5)$ and $\mathfrak{P}_{n}^{\prime}$ $(n=2,3,4)$ can be found in Appendix C. They yield a relation as

$\theta_{2}^{+}-\theta_{2}^{-}=\mathfrak{s} \sum_{n=0}^{4} \mathfrak{s}_{n} \bar{\alpha}^{n}$,

where the factor $\mathfrak{s}$ and the first two coefficients $\mathfrak{s}_{1}$ and $\mathfrak{s}_{2}$ are

$$
\begin{aligned}
\mathfrak{s} & =\frac{|\beta|}{(1+8 s \bar{\alpha})^{3}}, \\
\mathfrak{s}_{0} & =8 D^{2}+\frac{225}{256} \pi^{2}-16, \\
\mathfrak{s}_{1} & =s\left(256 D^{2}+\frac{2025}{64} \pi^{2}-\frac{2816}{5}\right) .
\end{aligned}
$$

The higher-order coefficients $\mathfrak{s}_{n}(n=2,3,4)$ can be found in Appendix C. 


\subsection{Magnification relations}

Using Eqs. (43)-(45) for magnifications and Eq. (60) for image positions, we can have

$$
\begin{aligned}
\mu_{0}^{ \pm}= & \frac{1}{2|\beta| \eta^{2}}\left[|\beta|\left(\beta^{2}+4\right) \pm\left(\beta^{2}+2\right) \eta\right. \\
& +16 s \bar{\alpha}(2|\beta| \pm \eta)], \\
\mu_{1}^{+}= & \mu_{1}^{-} \\
= & -\frac{15}{16} \pi \frac{1+18 s \bar{\alpha}+10 s \bar{\alpha}^{2}(15 s-2)}{\eta^{3}}
\end{aligned}
$$

and

$$
\mu_{2}^{ \pm}= \pm \mathfrak{M} \sum_{n=0}^{4} \mathfrak{M}_{n} \bar{\alpha}^{n},
$$

where the factor $\mathfrak{M}$ and the first two coefficients $\mathfrak{M}_{0}$ and $\mathfrak{M}_{1}$ are

$$
\begin{aligned}
\mathfrak{M}= & \frac{1}{|\beta| \eta^{5}}, \\
\mathfrak{M}_{0}= & \frac{8}{3} D^{2} \beta^{4}+\left(\frac{176}{3} D^{2}-32 D-32\right) \beta^{2} \\
& -128 D+192 D^{2}+\frac{675}{128} \pi^{2}-128, \\
\mathfrak{M}_{1}= & s\left[\frac{128}{3} D^{2} \beta^{4}+\left(1408 D^{2}-768 D-\frac{4352}{5}\right) \beta^{2}\right. \\
& \left.+6144 D^{2}-4096 D+\frac{6075}{32} \pi^{2}-\frac{22528}{5}\right] .
\end{aligned}
$$

The higher-order coefficients $\mathfrak{M}_{n}(n=2,3,4)$ can be found in Appendix C. They can give three simple magnification relations as

$$
\begin{aligned}
& \mu_{0}^{+}+\mu_{0}^{-}=1, \\
& \mu_{1}^{+}-\mu_{1}^{-}=0, \\
& \mu_{2}^{+}+\mu_{2}^{-}=0 .
\end{aligned}
$$

Again, the sign of the magnification denotes the parity of an image so that the absolute value of $\mu$ indicate its brightness. At the zeroth-order, the difference between the fluxes of images, $\left|\mu_{0}^{+}\right|-\left|\mu_{0}^{-}\right|$, equals to the flux of the source without lensing. The relation (83) emerges because both images have the same $\mu_{1}$. These relations are immune to the coupling to the Weyl tensor.

By combining Eqs. (33), (36), (43) and (44), we can verify that

$\mu_{0}^{+} \theta_{1}^{+}+\mu_{0}^{-} \theta_{1}^{-}+\mu_{1}^{+} \theta_{0}^{+}+\mu_{1}^{-} \theta_{0}^{-}=0$.

\subsection{Total magnification and centroid}

If the two images can not be separated, the observables are the total magnification and magnification-weighted centroid position. The relations about magnifications (82)-(84) leads to the total magnification as

$$
\begin{aligned}
\mu_{\mathrm{tot}} & =\left|\mu^{+}\right|+\left|\mu^{-}\right| \\
& =\left(2 \mu_{0}^{+}-1\right)+2 \epsilon^{2} \mu_{2}^{+}+\mathscr{O}\left(\epsilon^{3}\right) .
\end{aligned}
$$

The exact cancellation between $\mu_{1}^{+}$and $\mu_{1}^{-}$[see Eq. (83)] guarantee that $\mu_{\text {tot }}$ does not have the $\mathscr{O}(\epsilon)$ term.

The magnification-weighted centroid position is defined by [52]

$\Theta_{\text {cent }}=\frac{\theta^{+}\left|\mu^{+}\right|-\theta^{-}\left|\mu^{-}\right|}{\left|\mu^{+}\right|+\left|\mu^{-}\right|}=\frac{\theta^{+} \mu^{+}+\theta^{-} \mu^{-}}{\mu^{+}-\mu^{-}}$.

With the results obtained in the previous parts of this section, it can be expanded in the series of $\epsilon$ as

$\Theta_{\text {cent }}=\Theta_{0}+\varepsilon \Theta_{1}+\varepsilon^{2} \Theta_{2}+\mathscr{O}\left(\varepsilon^{3}\right)$,

where the zeroth-order, first-order and second-order terms are

$\Theta_{0}=|\beta| \frac{\beta^{2}+3+24 s \bar{\alpha}}{\beta^{2}+2+16 s \bar{\alpha}}$,

$\Theta_{1}=0$,

$\Theta_{2}=\mathfrak{S} \sum_{n=0}^{4} \mathfrak{S}_{n} \bar{\alpha}^{n}$.

In the expression of $\Theta_{2}$, the factor $\mathfrak{S}$ and the first two coefficients $\mathfrak{S}_{0}$ and $\mathfrak{S}_{1}$ are

$$
\begin{aligned}
\mathfrak{S}= & \frac{|\beta|}{\eta^{2}\left(\beta^{2}+2+16 s \bar{\alpha}\right)^{2}}, \\
\mathfrak{S}_{0}= & \frac{8}{3} D^{2} \beta^{6}+\left(\frac{104}{3} D-16\right) D \beta^{4}+\left(\frac{272}{3} D^{2}\right. \\
& -64 D+32) \beta^{2}-\frac{64}{3} D^{2}-\frac{675}{128} \pi^{2}+128, \\
\mathfrak{S}_{1}= & s\left[\frac{64}{3} D^{2} \beta^{6}+\left(\frac{1664}{3} D-256\right) D \beta^{4}\right. \\
& +\left(2176 D^{2}-1536 D+\frac{4352}{5}\right) \beta^{2} \\
& \left.-\frac{2048}{3} D^{2}-\frac{6075}{32} \pi^{2}+\frac{22528}{5}\right],
\end{aligned}
$$

and the higher-order coefficients $\mathfrak{S}_{n}(n=2,3,4)$ can be found in Appendix C.

\subsection{Differential time delay}

The differential delay between the positive- and negativeparity images is

$\Delta \hat{\tau}=\hat{\tau}_{-}-\hat{\tau}_{+}$, 
and it has a series form as

$$
\Delta \hat{\tau}=\Delta \hat{\tau}_{0}+\varepsilon \Delta \hat{\tau}_{1}+\mathscr{O}\left(\varepsilon^{2}\right),
$$

where the zeroth-order and first-order terms are

$$
\begin{aligned}
\Delta \hat{\tau}_{0}= & \frac{1}{2} \eta|\beta|+\ln \left(\frac{\eta+|\beta|}{\eta-|\beta|}\right), \\
\Delta \hat{\tau}_{1}= & \frac{15}{8} \pi \frac{|\beta|}{(1+8 s \bar{\alpha})^{2}}\left[\frac{1}{2}+11 s \bar{\alpha}\right. \\
& \left.+s \bar{\alpha}^{2}(111 s-2)+48 s^{2} \bar{\alpha}^{3}(15 s-2)\right] .
\end{aligned}
$$

Since both of them are affected by the coupling to the Weyl tensor, it is theoretically possible to test it by observing the differential time delay between two images (see next section for discussion).

\section{Observational effects}

By using the lensing relations found in the previous section, we can obtain the practical observables for the photons coupling to the Weyl tensor in the Schwarzschild black hole. After that, like Ref. [53], we will consider and discuss several astrophysical scenarios.

\subsection{Practical observables}

In order to proceed the investigation, we focus on the zerothorder and first-order lensing effects. The former ones are the observables of the weak deflection limits, and the latter ones might be able to be measured in the near future. To fulfill this purpose, we need convert the scaled variables $(\beta, \theta, \mu, \hat{\tau})$ to practical observables $(\mathscr{B}, \vartheta, F, \tau)$. Observables of lensing usually are the positions, fluxes and time delays of the images. The fluxes are connected to the magnifications through the flux of the source, i.e., $F_{i}=\left|\mu_{i}\right| F_{\text {src }}$.

Following the discussion in Ref. [53], we also construct some possibly measurable combinations of observables which are

$$
\begin{aligned}
P_{\mathrm{tot}} \equiv & \vartheta^{+}+\vartheta^{-} \\
= & \mathscr{E}+\frac{15}{16} \varepsilon \pi \vartheta_{\mathrm{E}} \frac{1+18 s \bar{\alpha}+10 s \bar{\alpha}^{2}(15 s-2)}{1+8 s \bar{\alpha}} \\
& +\mathscr{O}\left(\varepsilon^{2}\right), \\
\Delta P \equiv & \vartheta^{+}-\vartheta^{-} \\
= & |\mathscr{B}|\left[1-\frac{15}{16} \varepsilon \pi \frac{\vartheta_{\mathrm{E}}}{\mathscr{E}} \frac{1+18 s \bar{\alpha}+10 s \bar{\alpha}^{2}(15 s-2)}{1+8 s \bar{\alpha}}\right] \\
& +\mathscr{O}\left(\varepsilon^{2}\right), \\
F_{\mathrm{tot}} \equiv & F^{+}+F^{-} \\
= & F_{\mathrm{src}} \frac{\mathscr{B}^{2}+2 \vartheta_{\mathrm{E}}^{2}+16 s \bar{\alpha} \vartheta_{\mathrm{E}}^{2}}{|\mathscr{B}| \mathscr{E}}+\mathscr{O}\left(\varepsilon^{2}\right)
\end{aligned}
$$

$$
\begin{aligned}
& \Delta F \equiv F^{+}-F^{-} \\
& =F_{\mathrm{src}}-F_{\mathrm{src}} \frac{15}{8} \varepsilon \pi \frac{\vartheta_{\mathrm{E}}^{3}}{\mathscr{E}^{3}}\left[1+18 s \bar{\alpha}+10 s \bar{\alpha}^{2}(15 s-2)\right] \\
& +\mathscr{O}\left(\varepsilon^{2}\right), \\
& S_{\text {cent }} \equiv \frac{\vartheta^{+} F^{-}-\vartheta^{-} F^{-}}{F_{\text {tot }}} \\
& =|\mathscr{B}| \frac{\mathscr{B}^{2}+3 \vartheta_{\mathrm{E}}^{2}+24 s \bar{\alpha} \vartheta_{\mathrm{E}}^{2}}{\mathscr{B}^{2}+2 \vartheta_{\mathrm{E}}^{2}+16 s \bar{\alpha} \vartheta_{\mathrm{E}}^{2}}+\mathscr{O}\left(\varepsilon^{2}\right), \\
& \Delta \tau=\frac{d_{\mathrm{L}} d_{\mathrm{S}}}{c d_{\mathrm{LS}}}\left\{\frac{1}{2}|\mathscr{B}| \mathscr{E}+\vartheta_{\mathrm{E}}^{2} \ln \left(\frac{\mathscr{E}+|\mathscr{B}|}{\mathscr{E}-|\mathscr{B}|}\right)\right. \\
& +\varepsilon \frac{15}{8} \pi \frac{\vartheta_{\mathrm{E}}|\mathscr{B}|}{(1+8 s \bar{\alpha})^{2}}\left[\frac{1}{2}+11 s \bar{\alpha}+s \bar{\alpha}^{2}(111 s-2)\right. \\
& \left.\left.+48 s^{2} \bar{\alpha}^{3}(15 s-2)\right]+\mathscr{O}\left(\varepsilon^{2}\right)\right\} \text {. }
\end{aligned}
$$

Here, we define that

$\mathscr{E}=\sqrt{\mathscr{B}^{2}+4 \vartheta_{\mathrm{E}}^{2}+32 s \bar{\alpha} \vartheta_{\mathrm{E}}^{2}}$.

These combinations of observables represent our results about weak deflection lensing for photons coupling to the Weyl tensor in the Schwarzschild black hole. We will investigate the effects of the coupling on these practical observables so that we define following indicators to demonstrate its contributions:

$$
\begin{aligned}
\delta P_{\text {tot }} & \equiv P_{\text {tot }}-P_{\text {tot }}(\bar{\alpha}=0), \\
\delta \Delta P & \equiv \Delta P-\Delta P(\bar{\alpha}=0), \\
\delta r_{\text {tot }} & \equiv 2.5 \log _{10}\left[\frac{F_{\text {tot }}}{F_{\text {tot }}(\bar{\alpha}=0)}\right], \\
\delta \Delta r & \equiv 2.5 \log _{10}\left[\frac{\Delta F}{\Delta F(\bar{\alpha}=0)}\right], \\
\delta S_{\text {cent }} & \equiv F_{\text {cent }}-F_{\text {cent }}(\bar{\alpha}=0), \\
\delta \Delta \tau & \equiv \Delta \tau-\Delta \tau(\bar{\alpha}=0),
\end{aligned}
$$

where the differences between fluxes are converted into magnitudes. Keeping the leading contributions, we can have their dominant terms as

$$
\begin{aligned}
\delta P_{\mathrm{tot}} & =\frac{16 \vartheta_{\mathrm{E}}}{\sqrt{\beta^{2}+4}} s \bar{\alpha}+\mathscr{O}\left(\varepsilon, \bar{\alpha}^{2}\right), \\
\delta \Delta P & =-\frac{15}{8} \varepsilon \pi \vartheta_{\mathrm{E}} \frac{|\beta|\left(5 \beta^{2}+12\right)}{\left(\beta^{2}+4\right)^{3 / 2}} s \bar{\alpha}+\mathscr{O}\left(\varepsilon^{2}, \bar{\alpha}^{2}\right), \\
\delta r_{\mathrm{tot}} & =\frac{80}{(\ln 10)\left(\beta^{2}+2\right)\left(\beta^{2}+4\right)} s \bar{\alpha}+\mathscr{O}\left(\varepsilon^{2}, \bar{\alpha}^{2}\right), \\
\delta \Delta r & =-\frac{225}{8 \ln 10} \varepsilon \pi \frac{3 \beta^{2}+4}{\left(\beta^{2}+4\right)^{5 / 2}} s \bar{\alpha}+\mathscr{O}\left(\varepsilon^{2}, \bar{\alpha}^{2}\right), \\
\delta S_{\mathrm{cent}} & =\frac{8|\beta|^{3} \vartheta_{\mathrm{E}}}{\left(\beta^{2}+2\right)^{2}} s \bar{\alpha}+\mathscr{O}\left(\varepsilon^{2}, \bar{\alpha}^{2}\right), \\
\delta \Delta \tau & =\frac{45 \frac{d_{\mathrm{L}} d_{\mathrm{S}}}{8} \varepsilon \pi|\beta| \vartheta_{\mathrm{ES}}^{2} s \bar{\alpha}+\mathscr{O}\left(\varepsilon^{2}, \bar{\alpha}^{2}\right) .}{c d_{\mathrm{LS}}}
\end{aligned}
$$


They imply that the polarization $s$ and the strength $\alpha$ for photons coupling to the Weyl tensor cannot be simultaneously determined at least based on its leading observational effects.

Before investigating some observational examples numerically, we need to specify the domain of the coupling constant $\alpha$. In order to ensure that a photon can continuously propagate outside the event horizon of a Schwarzschild black hole, $\alpha$ has to satisfy two theoretical bounds $[46,47]$ that

$$
\begin{aligned}
& \alpha<\alpha_{+1}^{\text {th }} \text { for } s=+1, \\
& \alpha>\alpha_{-1}^{\text {th }} \text { for } s=-1,
\end{aligned}
$$

with

$\alpha_{+1}^{\text {th }}=m_{\bullet}^{2}=2.2 \times 10^{6}\left(\frac{M_{\bullet}}{M_{\odot}}\right)^{2} \mathrm{~m}^{2}$,

$\alpha_{-1}^{\text {th }}=-\frac{1}{2} m_{\bullet}^{2}=-1.1 \times 10^{6}\left(\frac{M_{\bullet}}{M_{\odot}}\right)^{2} \mathrm{~m}^{2}$.

where $M_{\bullet}$ and $M_{\odot}$ are the mass of the hole and the Sun. Based on experiments in the Solar System, an observational bound was found as [57]

$|s \alpha| \lesssim \alpha_{ \pm 1}^{\mathrm{obs}}=4 \times 10^{11} \mathrm{~m}^{2}$.

Thus, if it is assumed that the strength of $\alpha$ is irrelevant to the mass of the lens, the observational bound $\alpha_{ \pm 1}^{\text {obs }}$ will be much tighter than the theoretical ones $\alpha_{ \pm 1}^{\text {th }}$ for a supermassive system with $M_{\bullet} \gtrsim 10^{6} M_{\odot}$ and $\alpha_{+1}^{\text {th }}$ will be much more stringent for a stellar system with $M_{\bullet} \lesssim 10 M_{\odot}$. Both of these two kinds of bounds will be adopted in the following parts.

\subsection{The supermassive black hole in the Galactic Center}

By monitoring stellar orbits in the Galactic Center, the mass and distance to the supermassive black hole Sgr A* was determined as $M_{\bullet}=4.28 \times 10^{6} M_{\odot}$ and $d_{\mathrm{L}}=8.32 \mathrm{kpc}$ [59]. Its gravitational radius is $m_{\bullet}=6.32 \times 10^{9} \mathrm{~m}=2.05 \times 10^{-7}$ $\mathrm{pc}$, whose angular radius is $5.08 \times 10^{-6}$ arcsecond (as) and whose time scale is $\tau_{\mathrm{E}}=84.3 \mathrm{~s}$.

We consider a source orbiting the $\mathrm{Sgr} \mathrm{A}^{*}$ with a distance $d_{\mathrm{LS}} \ll d_{\mathrm{L}}$ so that $d_{\mathrm{S}} \approx d_{\mathrm{L}}$. We also define a scaled distance $d_{\mathrm{LS}}^{*}=d_{\mathrm{LS}} /(1 \mathrm{pc})$. From the perspective of the observer, if the source can be close enough to Sgr A* in the sky, it can be strongly lensed. The angular Einstein radius is $\vartheta_{\mathrm{E}}=$ $0.0224\left(d_{\mathrm{LS}}^{*}\right)^{1 / 2}$ as and the perturbation parameter is $\epsilon=$ $2.26 \times 10^{-4}\left(d_{\mathrm{LS}}^{*}\right)^{-1 / 2}$.

Therefore, based on Eq. (99), we can make a rough estimation, in which the first-order correction is dropped since it is smaller by 4 orders of magnitude, and find that the angular separation of the two lensed images is larger than

$P_{\text {tot, } \min }=0.0448\left(d_{\mathrm{LS}}^{*}\right)^{1 / 2} \sqrt{1+32 s \bar{\alpha}}$ as.
When the weak deflection lensing is considered that $b \gg m_{\bullet}$, $P_{\text {tot, min }}$ even for a source with $d_{\mathrm{LS}}^{*} \sim 10^{-3}$ (and resulting $\varepsilon \sim$ $7.16 \times 10^{-3}$ ) is still larger than the current resolution as low as 50 microarcsecond ( $\mu$ as) achieved by phase referencing optical/infrared interferometry [60]. It means that $P_{\text {tot }}, \Delta P$, $F_{\text {tot }}, \Delta F$ and $\Delta \tau$ can be obtained according to the positions, flux and timing of two separated images.

As a case study, we consider a source at $d_{\mathrm{LS}}=10^{-3}$ pc with its $\beta$ ranging from $10^{-3}$ to 10 . We take a wider domain of $\alpha$ belonging to $\left[10^{5}, 10^{20}\right] \mathrm{m}^{2}$ which can cover both $\alpha_{ \pm 1}^{\text {obs }}$ and $\left|\alpha_{ \pm 1}^{\text {th }}\right|$ for Sgr A*. It can be checked that $\bar{\alpha} \lesssim$ $1.2 \times 10^{-4}$ for both $s=1$ and -1 by using the relation of $\sin \vartheta=b / d_{\mathrm{L}}$ and the series solutions to the image positions. Our interest is the contributions on the lensing observables due to $\bar{\alpha}$. Estimations [see Eqs. (112)-(117)] suggest when $\bar{\alpha}$ is sufficiently small that is satisfied for this case, $s$ solely changes the sign of the leading effects of $\bar{\alpha}$. Therefore, we focus on the leading contributions for $s=1$, which can nicely approximate the results for $s=-1$ after changing their signs.

Figure 1 shows color-indexed $\delta P_{\text {tot }}, \delta \Delta P, \delta r_{\text {tot }}, \delta \Delta r_{\text {tot }}$ and $\delta \Delta \tau$ against $\alpha$ and $\beta$ in logarithmic scales. The coupling constant $\alpha$ can make the total separation of two images larger than its value in GR, i.e., $\delta P_{\text {tot }}>0$. When $\alpha$ is around $\alpha_{+1}^{\text {th }}$, $\delta P_{\text {tot }}$ can reach the level of $0.1 \mu$ as, which is still beyond the current ability. $\delta P_{\text {tot }}$ decreases when $\alpha$ drops; and if $\alpha \sim \alpha_{ \pm 1}^{\text {obs }}$, $\delta P_{\text {tot }}$ will be down to $\sim 10^{-9} \mu$ as far beyond the capability today. The existence of $\alpha$ can cause the angular difference between two images become smaller than the one in GR, i.e., $\delta \Delta P<0$. The sub-figure of $-\delta \Delta P$ has a similar pattern with its most significant contribution at $10^{-3} \mu$ as at $\alpha \sim \alpha_{+1}^{\text {th }}$. The effects of $\alpha$ on $\delta P_{\text {tot }}$ and $\delta \Delta P$ for Sgr $\mathrm{A}^{*}$ are too small to detect in the near future.

The total brightness of two images can be enhanced under the coupling to the Weyl tensor with respect to the one in GR and its difference $\delta r_{\text {tot }}$ can reach the level of $\sim 5 \times 10^{-4}$ mag, corresponding to relative flux of about 500 parts per million. Although it is within the current photometric accuracy of a dedicated space telescope, such as the Kepler mission for searching transiting exoplanets [61], the emission of Sgr $\mathrm{A}^{*}$ is constantly changing and its variable flux might easily overwhelm this variation of the total flux of two images due to the coupling. But it might still be a promising way to constrain $\alpha$ by measuring $\delta r_{\text {tot }}$ in the future if this noise can be well understood and separated. Additionally, the coupling can reduce the brightness difference between two images, but such as reduction is less than $\sim 10^{-6}$ mag below the current threshold. Finally, effects of $\alpha$ on the differential time delay between two images are represented. When $\alpha \sim \alpha_{+1}^{\text {th }}, \delta \Delta \tau$ can reach the level of $\sim 10^{-4} \mathrm{~s}$ which is practically inaccessible because the exposure time for astronomical imaging is usually much longer than it. 

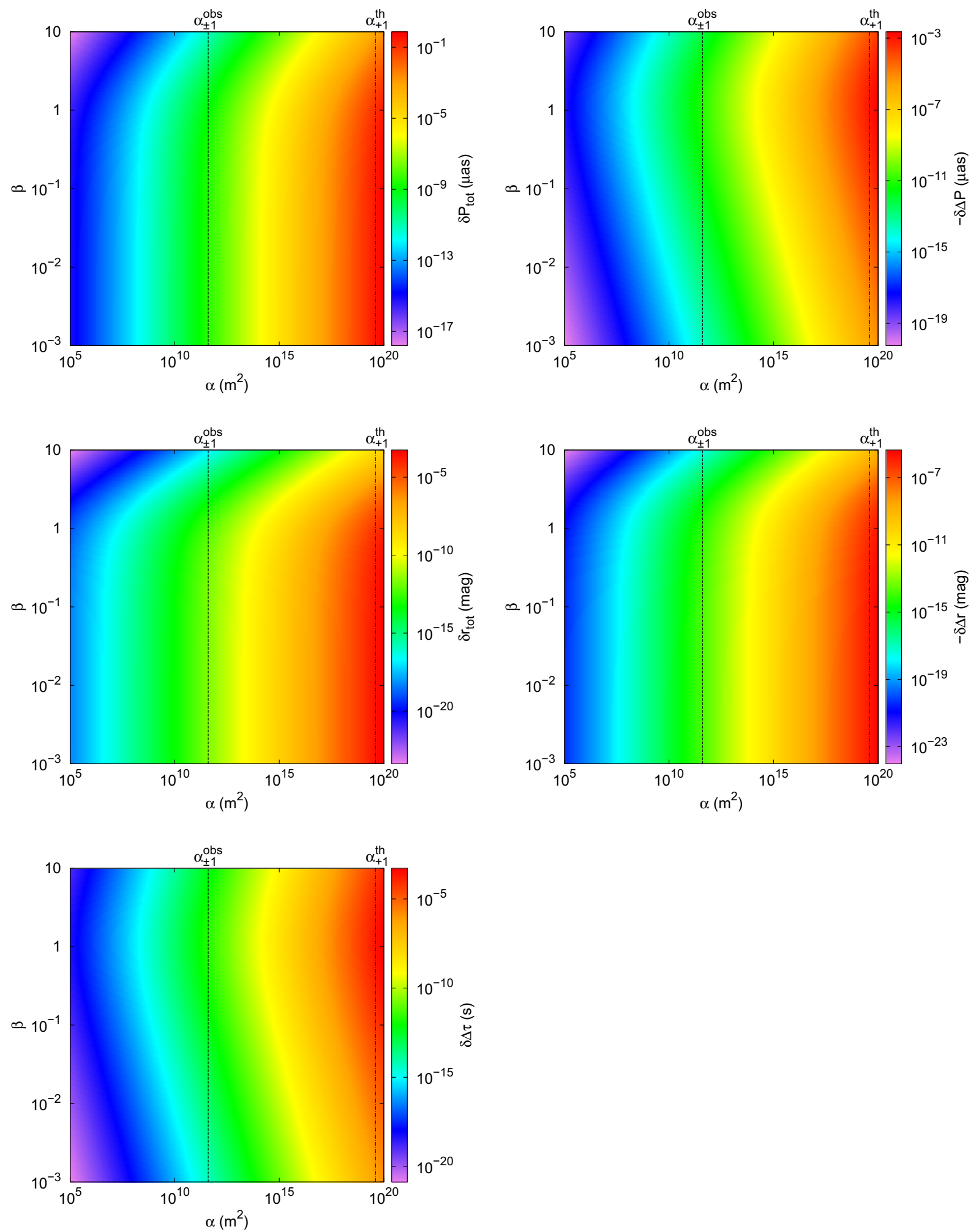

Fig. 1 Estimated contributions in observables of the weak deflection gravitational lensing caused by photons coupling to the Weyl tensor in the Schwarzschild black hole. We consider Sgr A* as the lens with the mass and distance as $M_{\bullet}=4.28 \times 10^{6} M_{\odot}$ and $d_{\mathrm{L}}=8.32 \mathrm{kpc}$ [59] and assume a source at a distance of $10^{-3} \mathrm{pc}$ from Sgr A*. From top to bottom panels and from left to right, color-indexed $\delta P_{\text {tot }}, \delta \Delta P, \delta r_{\text {tot }}$,

$\delta \Delta r_{\text {tot }}$ and $\delta \Delta \tau$ against $\alpha$ and $\beta$ are respectively presented in logarithmic scales. In each sub-figure, the dashed line denotes the observational bound on $\alpha$ and the dash-dot one shows the theoretical bound. Here, the polarization $s=1$ is taken; the contributions of $s=-1$ can be sufficiently approximated by changing the signs of the ones shown in each sub-figures 


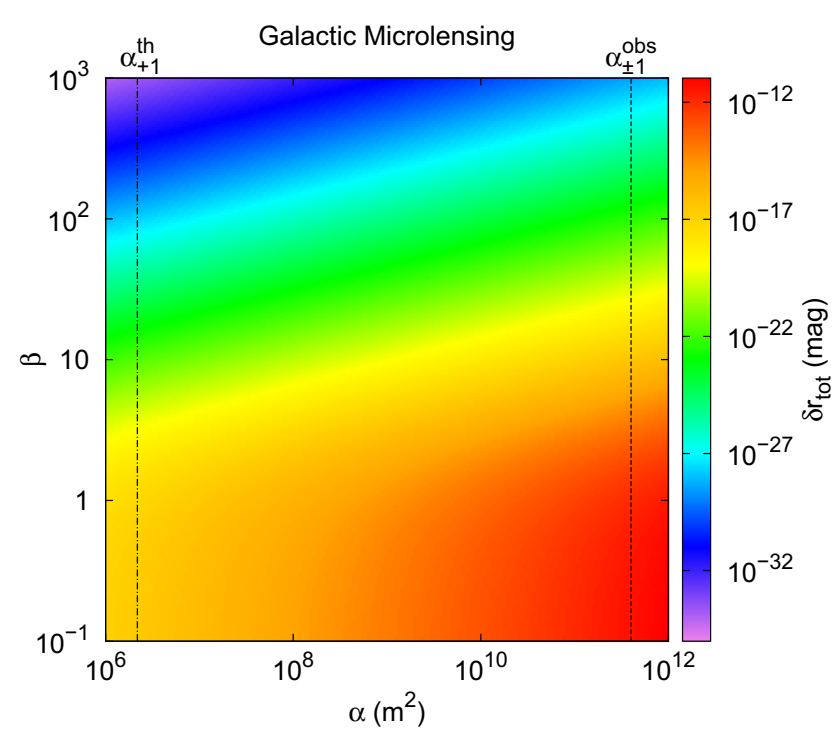

Fig. 2 Estimated contributions in microlensing caused by photons coupling to the Weyl tensor in the Schwarzschild black hole. Left: $\delta r_{\text {tot }}$ is shown for a Galactic lensing with $M_{\bullet}=M_{\odot}$ and $2 d_{\mathrm{L}}=d_{\mathrm{S}}=8 \mathrm{kpc}$. Right: $\delta S_{\text {cent }}$ is represented for an astrometric lensing with $M_{\bullet}=0.676$

In a summary, based on the current limit of the technology and the specific circumstance of the Sgr A*, the impact of the photons coupling to the Weyl tensor in the Schwarzschild black hole is unable to be detected in the observables of weak deflection lensing, while measuring on the variation of the total flux of two images might be a promising way for testing such a coupling in the future.

\subsection{Galactic and astrometric microlensing}

In a scenario of microlensing, a foreground gravitational object lenses a more distant background star. Plenty of projects [62-67] on Galactic microlensing measure change of the total fluxes of stars in the bulge with time, while astrometric microlensing on centroid shifts of a remote source by a nearby lens is also discussed [68-70] and practiced [71-73].

For Galactic lensing, masses and distances of the lens and source are respectively scaled by the solar mass and by 8 $\mathrm{kpc}$ as $M_{\bullet}^{*}=M_{\bullet} / M_{\odot}$ and $d_{\mathrm{S}}^{*}=d_{\mathrm{S}} /(8 \mathrm{kpc})$. We suppose a situation that the lens is located at rough midpoint between the observer and the source, i.e. $d_{\mathrm{L}} \sim d_{\mathrm{LS}} \sim d_{\mathrm{S}} / 2$. We can, therefore, have that $\vartheta_{\bullet} \sim 2.5 \times 10^{-12}\left(M_{\bullet}^{*} / d_{\mathrm{S}}^{*}\right)$ as, $\vartheta_{\mathrm{E}} \sim 10^{-3}\left(M_{\bullet}^{*} / d_{\mathrm{S}}^{*}\right)^{1 / 2}$ as and $\varepsilon \sim 2.4 \times 10^{-9}\left(M_{\bullet}^{*} / d_{\mathrm{S}}^{*}\right)^{1 / 2}$. We concentrate on the contribution of the coupling to the Weyl tensor on $F_{\text {tot }}$ which is indicated by $\delta r_{\text {tot }}$.

For astrometric lensing, masses and distances of the lens and source are respectively scaled by the solar mass and by $2 \mathrm{kpc}$ as $M_{\bullet}^{*}=M_{\bullet} / M_{\odot}$ and $d_{\mathrm{S}}^{*}=d_{\mathrm{S}} /(2 \mathrm{kpc})$. We assume that a nearby lens is located at $10 \mathrm{pc}$ from the observer, i.e. $d_{\mathrm{L}}^{*}=d_{\mathrm{L}} /(10 \mathrm{pc})$, so that $d_{\mathrm{S}} \approx d_{\mathrm{LS}}$. We can have that $\vartheta \bullet \sim$

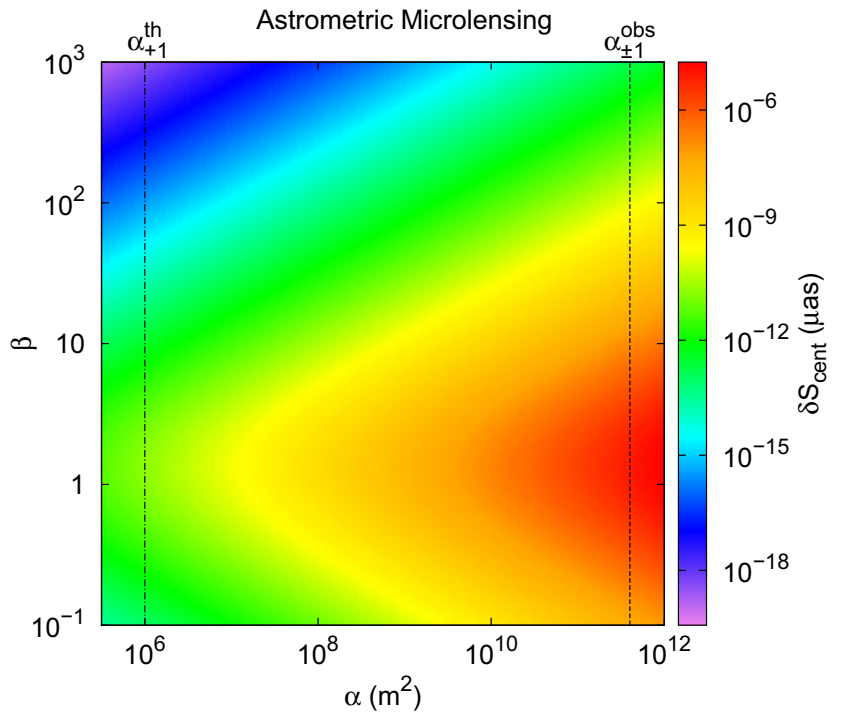

$M_{\odot}, d_{\mathrm{L}}=5.55 \mathrm{pc}$ and $d_{\mathrm{S}}=2 \mathrm{kpc}$. In each panels, the dashed line denotes the observational bound on $\alpha$ and the dash-dot one shows the theoretical bound, where the polarization $s=1$ is taken

$1.0 \times 10^{-9}\left(M_{\bullet}^{*} / d_{\mathrm{L}}^{*}\right)$ as, $\vartheta_{\mathrm{E}} \sim 2.9 \times 10^{-2}\left(M_{\bullet}^{*} / d_{\mathrm{L}}^{*}\right)^{1 / 2}$ as and $\varepsilon \sim 3.5 \times 10^{-8}\left(M_{\bullet}^{*} / d_{\mathrm{L}}^{*}\right)^{1 / 2}$. Astrometric lensing by a nearby star has an Einstein ring radius almost 30 times larger then the one of Galactic lensing. The centroid position $S_{\text {cent }}$ is the observables, in which $\delta S_{\text {cent }}$ indicates the contributions of $\alpha$.

The left panel of Fig. 2 shows color-indexed $\delta r_{\text {tot }}$ for a specific case of Galactic lensing, where we assume that $M_{\bullet}=M_{\odot}$ and $2 d_{\mathrm{L}}=d_{\mathrm{S}}=8 \mathrm{kpc}$. The coupling constant $\alpha$ is set belonging to the domain $\left[10^{6}, 10^{12}\right] \mathrm{m}^{2}$, covering both $\alpha_{ \pm 1}^{\text {obs }}$ (dashed line) and $\alpha_{+1}^{\text {th }}$ (dash-dot line) with $s=1$ for the lens. The right panel of Fig. 2 represents color-indexed $\delta S_{\text {cent }}$ for a case of astrometric lensing with $M_{\bullet}=0.676 M_{\odot}$, $d_{\mathrm{L}}=5.55 \mathrm{pc}$ and $d_{\mathrm{S}}=2 \mathrm{kpc}$ based on the microlensing event caused by a near white dwarf [73]. We take $\alpha \in\left[10^{5.5}, 10^{12}\right]$ with $s=1$ in order to contain the observational and theoretical bounds. However, the contributions of $\alpha$ in these two microlensing $\delta r_{\text {tot }}$ and $\delta S_{\text {cent }}$ are far beyond the current observational limits. The contributions from the other polarization for the coupling, i.e. $s=-1$, can be sufficiently approximated by changing the signs of the ones shown in this figure; and they are too small to detect as well.

\section{Conclusions and discussion}

In order to provide one of the missing puzzles of the whole physical picture of photons coupled to Weyl tensor in a Schwarzschild black hole, we investigate its weak deflection lensing, as an extension of the previous works on its 
strong deflection lensing $[46,47]$. Under a unified framework valid for both two polarization directions of the coupling, we obtain its bending angle, image position, magnification, centroid and time delay in the coordinate-invariant forms upon to the second order in the perturbation parameter of the ratio of the angular gravitational radius to angular Einstein radius of the lens. The contributions of such a coupling on some astrophysical scenarios are also studied. We find that, in the weak deflection lensing on a star orbiting the Sgr A*, Galactic microlensing on a star in the bulge and astrometric microlensing by a nearby lens, these effects caused by coupling are beyond the current limits of technology. However, measuring the variation of the total flux of two images caused by the Sgr A* might be a promising way for testing such a coupling in the future.

In this work, as an astrophysically important ingredient, the spin of a black hole has not been taken into account. A self-consistent treatment should move from the present model we considered to photons coupled to the Weyl tensor in a Kerr black hole, in which the strong deflection lensing was studied [48]. As expected, the spin can make the light propagation more complicated and effectively causes the caustic shifted and distorted [74]. We will leave the detailed investigation on its weak deflection lensing for future works.

Acknowledgements This work is funded by the National Natural Science Foundation of China (Grant No. 11573015).

Open Access This article is distributed under the terms of the Creative Commons Attribution 4.0 International License (http://creativecomm ons.org/licenses/by/4.0/), which permits unrestricted use, distribution, and reproduction in any medium, provided you give appropriate credit to the original author(s) and the source, provide a link to the Creative Commons license, and indicate if changes were made.

Funded by SCOAP ${ }^{3}$.

\section{Appendix A: Higher-order coefficients of $\mathfrak{b}_{n}, \mathfrak{a}_{n}$ and $\hat{\alpha}_{n}$}

In Eq. (11) for relation between $r_{0}$ and $b$, the higher-order coefficients $\mathfrak{b}_{n}(n=3, \ldots, 6)$ are

$$
\begin{aligned}
\mathfrak{b}_{3}= & 4+144 s \bar{\alpha}+288 s \bar{\alpha}^{2}(9 s-1) \\
& +768 s \bar{\alpha}^{3}\left(24 s^{2}-6 s+1\right), \\
\mathfrak{b}_{4}= & \frac{105}{8}+630 s \bar{\alpha}+756 s \bar{\alpha}^{2}(21 s-2) \\
& +864 s \bar{\alpha}^{3}\left(243 s^{2}-54 s+8\right) \\
& +96 s \bar{\alpha}^{4}\left(11979 s^{3}-4356 s^{2}+1188 s-80\right), \\
\mathfrak{b}_{5}= & 48+2880 s \bar{\alpha}+7680 s \bar{\alpha}^{2}(12 s-1) \\
& +23040 s \bar{\alpha}^{3}\left(75 s^{2}-15 s+2\right) \\
& +18432 s \bar{\alpha}^{4}\left(972 s^{3}-324 s^{2}+81 s-5\right) \\
& +\frac{6144}{5} s \bar{\alpha}^{5}\left(64827 s^{4}-30870 s^{3}+11025 s^{2}\right. \\
& -1470 s+88),
\end{aligned}
$$

$$
\begin{aligned}
\mathfrak{b}_{6}= & \frac{3003}{16}+\frac{27027}{2} s \bar{\alpha}+19305 s \bar{\alpha}^{2}(27 s-2) \\
& +34320 s \bar{\alpha}^{3}\left(363 s^{2}-66 s+8\right) \\
& +9360 s \bar{\alpha}^{4}\left(19773 s^{3}-6084 s^{2}+1404 s-80\right) \\
& +1152 s \bar{\alpha}^{5}\left(1366875 s^{4}-607500 s^{3}\right. \\
& \left.+202500 s^{2}-25200 s+1408\right) \\
& +\frac{768}{5} s \bar{\alpha}^{6}\left(38336139 s^{5}-22550670 s^{4}\right. \\
& \left.+9727740 s^{3}-1907400 s^{2}+217056 s-8960\right)
\end{aligned}
$$

In Eq. (17) for the deflection angle, the higher-order coefficients $\mathfrak{a}_{n}(n=3, \ldots, 6)$ are

$$
\begin{aligned}
& \mathfrak{a}_{3}=\frac{122}{3}-\frac{15}{2} \pi-s \bar{\alpha}_{0}\left(360 \pi-\frac{6624}{5}\right) \\
& -s \bar{\alpha}_{0}^{2}\left[\left(6615 \pi-\frac{794592}{35}\right) s-450 \pi+\frac{67008}{35}\right] \\
& -s \bar{\alpha}_{0}^{3}\left[\left(40500 \pi-\frac{4920064}{35}\right) s^{2}\right. \\
& \left.+\left(\frac{887296}{35}-5400 \pi\right) s-\frac{524288}{105}\right] \text {, } \\
& \mathfrak{a}_{4}=\frac{3465}{64} \pi-130+s \bar{\alpha}_{0}\left(\frac{20925}{8} \pi-7640\right) \\
& +s \bar{\alpha}_{0}^{2}\left[\left(\frac{2193345}{32} \pi-\frac{1042272}{5}\right) s+\frac{72768}{5}\right. \\
& \left.-\frac{81405}{16} \pi\right]+s \bar{\alpha}_{0}^{3}\left[\left(\frac{7112853}{8} \pi-\frac{95704704}{35}\right) s^{2}\right. \\
& \left.+\left(\frac{15065856}{35}-\frac{582957}{4} \pi\right) s+18711 \pi-\frac{1680384}{35}\right] \\
& +s \bar{\alpha}_{0}^{4}\left[\left(\frac{137971917}{32} \pi-\frac{461693952}{35}\right) s^{3}\right. \\
& +\left(\frac{107440128}{35}-\frac{8560647}{8} \pi\right) s^{2}+\left(\frac{2091231}{8} \pi\right. \\
& \left.\left.-\frac{21454848}{35}\right) s-\frac{38115}{2} \pi\right] \text {, } \\
& \mathfrak{a}_{5}=\frac{7783}{10}-\frac{3465}{16} \pi-s \bar{\alpha}_{0}\left(\frac{31185}{2} \pi-50724\right) \\
& -s \bar{\alpha}_{0}^{2}\left[\left(\frac{2230245}{4} \pi-1775920\right) s+\frac{354592}{3}\right. \\
& \left.-\frac{72525}{2} \pi\right]-s \bar{\alpha}_{0}^{3}\left[\left(\frac{44293365}{4} \pi-\frac{1927205376}{55}\right) s^{2}\right. \\
& +\left(\frac{299910144}{55}-\frac{3407805}{2} \pi\right) s+192870 \pi \\
& \left.-\frac{35034112}{55}\right]-s \bar{\alpha}_{0}^{4}\left[\left(\frac{899497791}{8} \pi\right.\right. \\
& \left.-\frac{1777650959232}{5005}\right) s^{3}+\left(\frac{433882040832}{5005}\right. \\
& \left.-\frac{54360801}{2} \pi\right) s^{2}+\left(\frac{11438253}{2} \pi\right.
\end{aligned}
$$




$$
\begin{aligned}
& \left.\left.-\frac{93408532992}{5005}\right) s+\frac{121436160}{143}-228690 \pi\right] \\
& -s \bar{\alpha}_{0}^{5}\left[\left(\frac{891827253}{2} \pi-\frac{35310191717376}{25025}\right) s^{4}\right. \\
& +\left(\frac{2292283060224}{5005}-142427646 \pi\right) s^{3} \\
& +\left(41789358 \pi-\frac{140029464576}{1001}\right) s^{2} \\
& +\left(\frac{66077966336}{5005}-3089880 \pi\right) s \\
& \left.-\frac{939524096}{975}\right] \text {, } \\
& \mathfrak{a}_{6}=\frac{310695}{256} \pi-\frac{21397}{6}+s \bar{\alpha}_{0}\left(\frac{25394985}{256} \pi-306390\right) \\
& +s \bar{\alpha}_{0}^{2}\left[\left(\frac{1107098685}{256} \pi-13502016\right) s\right. \\
& \left.+804768-\frac{33315975}{128} \pi\right]+s \bar{\alpha}_{0}^{3}\left[\left(\frac{7260774525}{64} \pi\right.\right. \\
& -355394880) s^{2}+\left(51735936-\frac{530614935}{32} \pi\right) s \\
& \left.+\frac{14503095}{8} \pi-5601792\right] \\
& +s \bar{\alpha}_{0}^{4}\left[\left(\frac{224621915265}{128} \pi-\frac{60538960512}{11}\right) s^{3}\right. \\
& +\left(\frac{71747670528}{55}-\frac{13344763365}{32} \pi\right) s^{2} \\
& +\left(\frac{2726718795}{32} \pi-\frac{14582128128}{55}\right) s \\
& \left.+\frac{125491200}{11}-\frac{30018825}{8} \pi\right] \\
& +s \bar{\alpha}_{0}^{5}\left[\left(\frac{3713261122953}{256} \pi-\frac{4140725045760}{91}\right) s^{4}\right. \\
& +\left(\frac{14750750029824}{1001}-\frac{301384679553}{64} \pi\right) s^{3} \\
& +\left(\frac{86997944619}{64} \pi-\frac{4236163098624}{1001}\right) s^{2} \\
& +\left(\frac{368270204928}{1001}-\frac{1933875405}{16} \pi\right) s \\
& \left.+\frac{10830105}{2} \pi-\frac{193527808}{13}\right] \\
& +s \bar{\alpha}_{0}^{6}\left[\left(\frac{12488303405007}{256} \pi-\frac{765525411606528}{5005}\right) s^{5}\right. \\
& +\left(\frac{307851253530624}{5005}-\frac{2521647706203}{128} \pi\right) s^{4} \\
& +\left(\frac{460902199923}{64} \pi-\frac{111457297907712}{5005}\right) s^{3} \\
& +\left(\frac{14186020405248}{5005}-\frac{30733018821}{32} \pi\right) s^{2}
\end{aligned}
$$

$$
\begin{aligned}
& +\left(\frac{725498163}{8} \pi-\frac{99346808832}{455}\right) s \\
& -4339335 \pi] .
\end{aligned}
$$

In Eq. (22) for the gauge-invariant form of the deflection angle, the higher-order coefficients $\hat{\alpha}_{n}(n=3, \ldots, 6)$ are

$$
\begin{aligned}
\hat{\alpha}_{3}= & \frac{128}{3}+\frac{6144}{5} s \bar{\alpha}+\frac{73728}{35} s(9 s-1) \bar{\alpha}^{2} \\
& +\frac{524288}{105} s\left(24 s^{2}-6 s+1\right) \bar{\alpha}^{3},
\end{aligned}
$$

$$
\begin{aligned}
\hat{\alpha}_{4}= & \pi\left[\frac{3465}{64}+\frac{17325}{8} s \bar{\alpha}+\frac{72765}{32} s \bar{\alpha}^{2}(21 s-2)\right. \\
& +\frac{18711}{8} s \bar{\alpha}^{3}\left(243 s^{2}-54 s+8\right)+\frac{7623}{32} s \bar{\alpha}^{4}\left(11979 s^{3}\right. \\
& \left.\left.-4356 s^{2}+1188 s-80\right)\right]
\end{aligned}
$$

$$
\begin{aligned}
\hat{\alpha}_{5}= & \frac{3584}{5}+36864 s \bar{\alpha}+\frac{262144}{3} s \bar{\alpha}^{2}(12 s-1) \\
& +\frac{2621440}{11} s \bar{\alpha}^{3}\left(75 s^{2}-15 s+2\right)
\end{aligned}
$$$$
+\frac{25165824}{143} s \bar{\alpha}^{4}\left(972 s^{3}-324 s^{2}+81 s-5\right)
$$$$
+\frac{117440512}{10725} s \bar{\alpha}^{5}\left(64827 s^{4}-30870 s^{3}+11025 s^{2}\right.
$$$$
-1470 s+88) \text {, }
$$

$$
\begin{aligned}
\hat{\alpha}_{6}= & \pi\left[\frac{255255}{256}+\frac{16081065}{256} s \bar{\alpha}+s \bar{\alpha}^{2}\left(\frac{558242685}{256} s\right.\right. \\
& \left.-\frac{20675655}{128}\right)+s \bar{\alpha}^{3}\left(\frac{3057699645}{64} s^{2}-\frac{277972695}{32} s\right. \\
& \left.+\frac{8423415}{8}\right)+s \bar{\alpha}^{4}\left(\frac{84359626065}{128} s^{3}\right. \\
& \left.-\frac{6489202005}{32} s^{2}+\frac{1497508155}{32} s-\frac{21332025}{8}\right) \\
& +s \bar{\alpha}^{5}\left(\frac{1345763615625}{256} s^{4}-\frac{149529290625}{64} s^{3}\right. \\
& \left.+\frac{49843096875}{64} s^{2}-\frac{1550674125}{16} s+\frac{10830105}{2}\right) \\
& +s \bar{\alpha}^{6}\left(\frac{4752952849359}{256} s^{5}-\frac{1397927308635}{128} s^{4}\right. \\
& +\frac{301513733235}{64} s^{3}-\frac{29560169925}{32} s^{2} \\
& \left.\left.+\frac{840963123}{8} s-4339335\right)\right]
\end{aligned}
$$

\section{Appendix B: Higher-order coefficients of $\mathfrak{p}_{n}, \mathfrak{m}_{n}$ and $T_{n}$}

In Eq. (37) for the second-order correction to the image position, the higher-order coefficients $\mathfrak{p}_{n}(n=2, \ldots, 5)$ are 


$$
\begin{aligned}
\mathfrak{p}_{2}= & s\left\{s \left[D\left(\frac{4096}{3} D-1024\right) \theta_{0}^{6}\right.\right. \\
& +\left(5632 D^{2}-6144 D+\frac{201728}{35}\right) \theta_{0}^{4} \\
& +\left(2048 D^{2}-6144 D+\frac{647168}{35}-\frac{8775}{8} \pi^{2}\right) \theta_{0}^{2} \\
& \left.+\frac{96256}{7}-\frac{10240}{3} D^{2}-\frac{12825}{16} \pi^{2}\right] \\
& -\frac{18432}{35} \theta_{0}^{4}+\left(\frac{1125}{16} \pi^{2}-\frac{36864}{35}\right) \theta_{0}^{2} \\
& \left.-\frac{18432}{35}+\frac{1125}{32} \pi^{2}\right\},
\end{aligned}
$$

$\mathfrak{p}_{3}=s\left\{s^{2}\left[\left(\frac{45056}{3} D^{2}-16384 D+\frac{3432448}{105}\right) \theta_{0}^{4}\right.\right.$

$+\left(\frac{32768}{3} D^{2}-32768 D+\frac{3309568}{21}-\frac{151875}{16} \pi^{2}\right) \theta_{0}^{2}$

$\left.-\frac{81920}{3} D^{2}+\frac{16039936}{105}-\frac{292275}{32} \pi^{2}\right]$

$+s\left[-\frac{262144}{35} \theta_{0}^{4}+\left(\frac{10125}{8} \pi^{2}-\frac{163840}{7}\right) \theta_{0}^{2}\right.$

$\left.+\frac{14625}{16} \pi^{2}-\frac{557056}{35}\right]$

$\left.+\frac{131072}{105} \theta_{0}^{4}+\frac{262144}{105} \theta_{0}^{2}+\frac{131072}{105}\right\}$

$\mathfrak{p}_{4}=s^{2}\left\{s^{2}\left[\left(\frac{65536}{3} D^{2}-65536 D+\frac{54919168}{105}\right.\right.\right.$

$\left.-\frac{1265625}{32} \pi^{2}\right) \theta_{0}^{2}-\frac{327680}{3} D^{2}+\frac{93650944}{105}$

$\left.-\frac{3695625}{64} \pi^{2}\right]+s\left[\left(\frac{84375}{8} \pi^{2}-\frac{4194304}{35}\right) \theta_{0}^{2}\right.$

$\left.+\frac{165375}{16} \pi^{2}-\frac{5373952}{35}\right]+\left(\frac{2097152}{105}\right.$

$\left.\left.-\frac{5625}{8} \pi^{2}\right) \theta_{0}^{2}+\frac{2097152}{105}-\frac{5625}{16} \pi^{2}\right\}$,

$\mathfrak{p}_{5}=s^{3}\left[s^{2}\left(-\frac{524288}{3} D^{2}+\frac{219676672}{105}-\frac{1265625}{8} \pi^{2}\right)\right.$

$+s\left(\frac{84375}{2} \pi^{2}-\frac{16777216}{35}\right)+\frac{8388608}{105}$

$\left.-\frac{5625}{2} \pi^{2}\right]$.

In Eq. (45) for the second-order correction to the magnification, the higher-order coefficients $\mathfrak{m}_{n}(n=2, \ldots, 6)$ are

$$
\begin{aligned}
\mathfrak{m}_{2}= & s\left\{s \left[\frac{512}{3} D^{2} \theta_{0}^{8}+\left(9216 D^{2}-6144 D\right.\right.\right. \\
& \left.-\frac{403456}{35}\right) \theta_{0}^{6}+\left(34816 D^{2}-24576 D\right. \\
& \left.+\frac{26325}{8} \pi^{2}-\frac{1294336}{35}\right) \theta_{0}^{4}+\left(30720 D^{2}\right.
\end{aligned}
$$

$$
\begin{aligned}
& \left.\left.-20480 D-\frac{192512}{7}\right) \theta_{0}^{2}+2560 D^{2}\right]+\frac{36864}{35} \theta_{0}^{6} \\
& \left.+\left(\frac{73728}{35}-\frac{3375}{16} \pi^{2}\right) \theta_{0}^{4}+\frac{36864}{35} \theta_{0}^{2}\right\} \text {, } \\
& \mathfrak{m}_{3}=s\left\{s ^ { 2 } \left[\left(24576 D^{2}-16384 D-\frac{6864896}{105}\right) \theta_{0}^{6}\right.\right. \\
& +\left(\frac{557056}{3} D^{2}-131072 D+\frac{455625}{16} \pi^{2}\right. \\
& \left.-\frac{6619136}{21}\right) \theta_{0}^{4}+\left(245760 D^{2}-163840 D\right. \\
& \left.\left.-\frac{32079872}{105}\right) \theta_{0}^{2}+\frac{81920}{3} D^{2}\right]+s \theta_{0}^{2}\left[\frac{524288}{35} \theta_{0}^{4}\right. \\
& \left.+\left(\frac{327680}{7}-\frac{30375}{8} \pi^{2}\right) \theta_{0}^{2}+\frac{1114112}{35}\right] \\
& \left.-\frac{262144}{105} \theta_{0}^{6}-\frac{524288}{105} \theta_{0}^{4}-\frac{262144}{105} \theta_{0}^{2}\right\} \\
& \mathfrak{m}_{4}=s^{2}\left\{s ^ { 2 } \left[\left(\frac{1114112}{3} D^{2}-262144 D+\frac{3796875}{32} \pi^{2}\right.\right.\right. \\
& \left.-\frac{109838336}{105}\right) \theta_{0}^{4}+\left(983040 D^{2}-655360 D\right. \\
& \left.\left.-\frac{187301888}{105}\right) \theta_{0}^{2}+163840 D^{2}\right]+s \theta_{0}^{2}\left[\left(\frac{8388608}{35}\right.\right. \\
& \left.\left.-\frac{253125}{8} \pi^{2}\right) \theta_{0}^{2}+\frac{10747904}{35}\right]+\left(\frac{16875}{8} \pi^{2}\right. \\
& \left.\left.-\frac{4194304}{105}\right) \theta_{0}^{4}-\frac{4194304}{105} \theta_{0}^{2}\right\} \\
& \mathfrak{m}_{5}=s^{3}\left\{s ^ { 2 } \left[\left(1572864 D^{2}-1048576 D-\frac{439353344}{105}\right) \theta_{0}^{2}\right.\right. \\
& \left.\left.+524288 D^{2}\right]+s \frac{33554432}{35} \theta_{0}^{2}-\frac{16777216}{105} \theta_{0}^{2}\right\} \text {, } \\
& \mathfrak{m}_{6}=\frac{2097152}{3} s^{6} D^{2} .
\end{aligned}
$$

In Eq. (53) for the time delay, the higher-order coefficients $T_{n}(n=2,3)$ are

$$
\begin{aligned}
T_{2}= & \frac{15}{4} \pi-\frac{15}{2} \arctan \left(\frac{\xi}{\sqrt{1-\xi^{2}}}\right)-\frac{\sqrt{1-\xi^{2}}}{(1+\xi)^{2}}\left(\frac{5}{2} \xi+2\right) \\
& +s \bar{\alpha}_{0}\left[45 \pi-90 \arctan \left(\frac{\xi}{\sqrt{1-\xi^{2}}}\right)\right. \\
& \left.+6 \frac{\sqrt{1-\xi^{2}}}{(1+\xi)^{2}}\left(3 \xi^{3}+4 \xi^{2}-13 \xi-12\right)\right] \\
& +s \bar{\alpha}_{0}^{2}\left\{s \left[\frac{675}{2} \pi-675 \arctan \left(\frac{\xi}{\sqrt{1-\xi^{2}}}\right)\right.\right. \\
& \left.+9 \frac{\sqrt{1-\xi^{2}}}{(1+\xi)^{2}}\left(2 \xi^{5}+4 \xi^{4}+21 \xi^{3}+22 \xi^{2}-69 \xi-64\right)\right] \\
& -45 \pi+90 \arctan \left(\frac{\xi}{\sqrt{1-\xi^{2}}}\right)
\end{aligned}
$$




$$
\begin{aligned}
& \left.-6 \xi\left(2 \xi^{2}+7\right) \sqrt{1-\xi^{2}}\right\} \\
& T_{3}=-\frac{15}{4} \pi+\frac{15}{2} \arctan \left(\frac{\xi}{\sqrt{1-\xi^{2}}}\right) \\
& +\frac{1}{2} \frac{\sqrt{1-\xi^{2}}}{(1+\xi)^{3}}\left(35 \xi^{3}+133 \xi^{2}+157 \xi+60\right) \\
& +s \bar{\alpha}_{0}\left[-180 \pi+360 \arctan \left(\frac{\xi}{\sqrt{1-\xi^{2}}}\right)+6 \frac{\sqrt{1-\xi^{2}}}{(1+\xi)^{3}}\right. \\
& \left.\times\left(5 \xi^{5}+12 \xi^{4}+60 \xi^{3}+244 \xi^{2}+316 \xi+128\right)\right] \\
& +s \bar{\alpha}_{0}^{2}\left\{s \left[-\frac{6615}{2} \pi+6615 \arctan \left(\frac{\xi}{\sqrt{1-\xi^{2}}}\right)\right.\right. \\
& +\frac{9}{5} \frac{\sqrt{1-\xi^{2}}}{(1+\xi)^{3}}\left(24 \xi^{7}+62 \xi^{6}+354 \xi^{5}+535 \xi^{4}\right. \\
& \left.\left.+2605 \xi^{3}+12239 \xi^{2}+16517 \xi+6784\right)\right]+225 \pi \\
& -450 \arctan \left(\frac{\xi}{\sqrt{1-\xi^{2}}}\right)-\frac{6}{5} \frac{\sqrt{1-\xi^{2}}}{(1+\xi)}\left(24 \xi^{5}+14 \xi^{4}\right. \\
& \left.\left.+102 \xi^{3}+17 \xi^{2}+489 \xi+944\right)\right\} \\
& +s \bar{\alpha}_{0}^{3}\left\{s ^ { 2 } \left[-20250 \pi+40500 \arctan \left(\frac{\xi}{\sqrt{1-\xi^{2}}}\right)\right.\right. \\
& +\frac{36}{35} \frac{\sqrt{1-\xi^{2}}}{(1+\xi)^{3}}\left(40 \xi^{9}+120 \xi^{8}+896 \xi^{7}+2158 \xi^{6}\right. \\
& +6466 \xi^{5}+8675 \xi^{4}+30105 \xi^{3}+131411 \xi^{2} \\
& +176273 \xi+72256)]+s[2700 \pi \\
& -5400 \arctan \left(\frac{\xi}{\sqrt{1-\xi^{2}}}\right)-\frac{72}{35} \frac{\sqrt{1-\xi^{2}}}{(1+\xi)}\left(40 \xi^{7}\right. \\
& +40 \xi^{6}+272 \xi^{5}+202 \xi^{4}+946 \xi^{3}+351 \xi^{2} \\
& +3887 \xi+7072)]+\frac{768}{35} \frac{\sqrt{1-\xi^{2}}}{(1+\xi)}\left(5 \xi^{7}+5 \xi^{6}\right. \\
& \left.\left.+13 \xi^{5}+13 \xi^{4}+29 \xi^{3}+29 \xi^{2}+93 \xi+128\right)\right\}
\end{aligned}
$$

$$
\begin{aligned}
\mathfrak{s}_{3}= & s\left[s^{2}\left(16384 D^{2}+\frac{151875}{32} \pi^{2}-\frac{1654784}{21}\right)\right. \\
& \left.+s\left(\frac{81920}{7}-\frac{10125}{16} \pi^{2}\right)-\frac{131072}{105}\right], \\
\mathfrak{s}_{4}= & s^{2}\left[s^{2}\left(32768 D^{2}+\frac{1265625}{64} \pi^{2}-\frac{27459584}{105}\right)\right. \\
& +s\left(\frac{2097152}{35}-\frac{84375}{16} \pi^{2}\right)+\frac{5625}{16} \pi^{2} \\
& \left.-\frac{1048576}{105}\right] .
\end{aligned}
$$

In Eq. (66) for the second-order correction to the positions of the positive- and negative-parity images, the higher-order coefficients $\mathfrak{P}_{n}(n=2, \ldots, 5)$ and $\mathfrak{P}_{n}^{\prime}(n=2,3,4)$ are

$$
\begin{aligned}
\mathfrak{P}_{2}= & s\left\{s \left[D\left(\frac{65536}{3} D-8192\right) \beta^{6}+\left(323584 D^{2}\right.\right.\right. \\
& \left.-196608 D+\frac{1613824}{35}\right) \beta^{4}+\left(1097728 D^{2}\right. \\
& \left.-884736 D+\frac{15532032}{35}-8775 \pi^{2}\right) \beta^{2} \\
& \left.+\frac{1638400}{3} D^{2}-655360 D+\frac{6160384}{7}-38475 \pi^{2}\right] \\
& -\frac{147456}{35} \beta^{4}+\left(\frac{1125}{2} \pi^{2}-\frac{884736}{35}\right) \beta^{2} \\
& \left.+\frac{3375}{2} \pi^{2}-\frac{1179648}{35}\right\}, \\
\mathfrak{P}_{3}= & s\left\{s ^ { 2 } \left[\left(\frac{2588672}{3} D^{2}-524288 D+\frac{27459584}{105}\right) \beta^{4}\right.\right. \\
& +\left(\frac{17563648}{3} D^{2}-4718592 D+\frac{26476544}{7}\right. \\
& \left.-\frac{151875}{2} \pi^{2}\right) \beta^{2}+\frac{13107200}{3} D^{2}-5242880 D \\
& \left.+\frac{1026555904}{105}-\frac{876825}{2} \pi^{2}\right]+s\left[-\frac{2097152}{35} \beta^{4}\right. \\
& +\left(10125 \pi^{2}-\frac{3932160}{7}\right) \beta^{2}+43875 \pi^{2} \\
& \left.-\frac{35651584}{35}\right]+\frac{1048576}{105} \beta^{4}+\frac{2097152}{35} \beta^{2} \\
& \left.+\frac{8388608}{105}\right\},
\end{aligned}
$$

\section{$\mathfrak{M}_{n}$ and $\mathfrak{S}_{n}$}

In Eq. (72) for the relation about the second-order corrections to the image positions, the higher-order coefficients $\mathfrak{s}_{n}(n=$ $2,3,4)$ are

$$
\begin{aligned}
\mathfrak{s}_{2}= & s\left[s\left(3072 D^{2}+\frac{8775}{16} \pi^{2}-\frac{323584}{35}\right)\right. \\
& \left.+\frac{18432}{35}-\frac{1125}{32} \pi^{2}\right],
\end{aligned}
$$

$$
\begin{aligned}
\mathfrak{P}_{4}= & s^{2}\left\{s ^ { 2 } \left[\left(\frac{35127296}{3} D^{2}-9437184 D+\frac{439353344}{35}\right.\right.\right. \\
& \left.-\frac{1265625}{4} \pi^{2}\right) \beta^{2}+\frac{52428800}{3} D^{2}-20971520 D \\
& \left.+\frac{5993660416}{105}-\frac{11086875}{4} \pi^{2}\right]+s\left[\left(84375 \pi^{2}\right.\right. \\
& \left.\left.-\frac{100663296}{35}\right) \beta^{2}-\frac{343932928}{35}+496125 \pi^{2}\right]
\end{aligned}
$$




$$
\begin{aligned}
& +\left(\frac{16777216}{35}-5625 \pi^{2}\right) \beta^{2}+\frac{134217728}{105} \\
& \left.-16875 \pi^{2}\right\}, \\
\mathfrak{P}_{5}= & s^{3}\left[s ^ { 2 } \left(\frac{83886080}{3} D^{2}-33554432 D-7593750 \pi^{2}\right.\right. \\
& \left.+\frac{14059307008}{105}\right)+s\left(2025000 \pi^{2}\right. \\
& \left.\left.-\frac{1073741824}{35}\right)+\frac{536870912}{105}-135000 \pi^{2}\right],
\end{aligned}
$$

$$
\begin{aligned}
\mathfrak{P}_{2}^{\prime}= & s\left\{s \left[D\left(\frac{57344}{3} D-8192\right) \beta^{4}\right.\right. \\
& +\left(217088 D^{2}-147456 D+\frac{1613824}{35}\right) \beta^{2} \\
& \left.+425984 D^{2}-393216 D+\frac{10354688}{35}-8775 \pi^{2}\right] \\
& \left.-\frac{147456}{35} \beta^{2}+\frac{1125}{2} \pi^{2}-\frac{589824}{35}\right\},
\end{aligned}
$$

$$
\mathfrak{P}_{3}^{\prime}=s\left\{s ^ { 2 } \left[\left(\frac{1736704}{3} D^{2}-393216 D+\frac{27459584}{105}\right) \beta^{2}\right.\right.
$$$$
+\frac{6815744}{3} D^{2}-2097152 D+\frac{52953088}{21}
$$$$
\left.-\frac{151875}{2} \pi^{2}\right]+s\left(-\frac{2097152}{35} \beta^{2}+10125 \pi^{2}\right.
$$$$
\left.\left.-\frac{2621440}{7}\right)+\frac{1048576}{105} \beta^{2}+\frac{4194304}{105}\right\}
$$

$$
\begin{aligned}
\mathfrak{P}_{4}^{\prime}= & s^{2}\left[s ^ { 2 } \left(\frac{13631488}{3} D^{2}-4194304 D+\frac{878706688}{105}\right.\right. \\
& \left.-\frac{1265625}{4} \pi^{2}\right)+s\left(84375 \pi^{2}-\frac{67108864}{35}\right) \\
& \left.+\frac{33554432}{105}-5625 \pi^{2}\right]
\end{aligned}
$$

In Eq. (78) for the second-order correction to the magnifications of the positive- and negative-parity images, the higher-order coefficients $\mathfrak{M}_{n}(n=2,3,4)$ are

$$
\begin{aligned}
\mathfrak{M}_{2}= & s\left\{s \left[\frac{512}{3} D^{2} \beta^{4}+\left(11264 D^{2}-6144 D-\frac{403456}{35}\right) \beta^{2}\right.\right. \\
& \left.+73728 D^{2}-49152 D+\frac{26325}{8} \pi^{2}-\frac{2588672}{35}\right] \\
& \left.+\frac{36864}{35} \beta^{2}-\frac{3375}{16} \pi^{2}+\frac{147456}{35}\right\}, \\
\mathfrak{M}_{3}= & s\left\{s ^ { 2 } \left[\left(\frac{90112}{3} D^{2}-16384 D-\frac{6864896}{105}\right) \beta^{2}\right.\right. \\
& \left.+393216 D^{2}-262144 D+\frac{455625}{16} \pi^{2}-\frac{13238272}{21}\right] \\
& +s\left(\frac{524288}{35} \beta^{2}+\frac{655360}{7}-\frac{30375}{8} \pi^{2}\right)
\end{aligned}
$$

$$
\begin{aligned}
& \left.-\frac{262144}{105} \beta^{2}-\frac{1048576}{105}\right\}, \\
\mathfrak{M}_{4}= & s^{2}\left[s ^ { 2 } \left(786432 D^{2}-524288 D+\frac{3796875}{32} \pi^{2}\right.\right. \\
& \left.-\frac{219676672}{105}\right)+s\left(\frac{16777216}{35}-\frac{253125}{8} \pi^{2}\right) \\
& \left.+\frac{16875}{8} \pi^{2}-\frac{8388608}{105}\right] .
\end{aligned}
$$

In Eq. (91) for the second-order correction to the magnification-weighted centroid position, the higher-order coefficients $\mathfrak{S}_{n}(n=2,3,4)$ are

$$
\begin{aligned}
\mathfrak{S}_{2}= & s\left[s \left(\frac{6656}{3} D^{2} \beta^{4}-1024 D \beta^{4}+17408 D^{2} \beta^{2}\right.\right. \\
& -12288 D \beta^{2}+\frac{403456}{35} \beta^{2}+\frac{2588672}{35}-8192 D^{2} \\
& \left.-\frac{26325}{8} \pi^{2}\right)-\frac{36864}{35} \beta^{2}-\frac{147456}{35} \\
& \left.+\frac{3375}{16} \pi^{2}\right], \\
\mathfrak{S}_{3}= & s\left[s ^ { 2 } \left(\frac{139264}{3} D^{2} \beta^{2}-32768 D \beta^{2}+\frac{6864896}{105} \beta^{2}\right.\right. \\
& \left.-\frac{131072}{3} D^{2}-\frac{455625}{16} \pi^{2}+\frac{13238272}{21}\right) \\
& +s\left(-\frac{524288}{35} \beta^{2}+\frac{30375}{8} \pi^{2}-\frac{655360}{7}\right) \\
& \left.+\frac{262144}{105} \beta^{2}+\frac{1048576}{105}\right], \\
& s^{2}\left[s^{2}\left(-\frac{262144}{3} D^{2}+\frac{219676672}{105}-\frac{3796875}{32} \pi^{2}\right)\right. \\
& +s\left(\frac{253125}{8} \pi^{2}-\frac{16777216}{35}\right)+\frac{8388608}{105} \\
\mathfrak{S}_{4} & \left.16875 \pi^{2}\right]
\end{aligned}
$$

\section{References}

1. P. Schneider, J. Ehlers, E.E. Falco, Gravitational Lenses (Springer, Berlin, 1992). https://doi.org/10.1007/978-3-662-03758-4

2. A.O. Petters, H. Levine, J. Wambsganss, Singularity Theory and Gravitational Lensing (Birkhäuser, Boston, 2001). https://doi.org/ 10.1007/978-1-4612-0145-8

3. P. Schneider, C.S. Kochanek, J. Wambsganss, in Saas-Fee Advanced Course 33: Gravitational Lensing: Strong, Weak and Micro, ed. by G. Meylan, P. Jetzer, P. North (Springer, Berlin, 2006). https://doi.org/10.1007/978-3-540-30310-7

4. V. Perlick, Living Rev. Relativ. 7, 9 (2004). https://doi.org/10. 12942/lrr-2004-9

5. I.T. Drummond, S.J. Hathrell, Phys. Rev. D 22, 343 (1980). https:// doi.org/10.1103/PhysRevD.22.343

6. L. Mankiewicz, M. Misiak, Phys. Rev. D 40, 2134 (1989). https:// doi.org/10.1103/PhysRevD.40.2134 
7. I.B. Khriplovich, Phys. Lett. B 346, 251 (1995). https://doi.org/10. 1016/0370-2693(94)01679-7

8. R.D. Daniels, G.M. Shore, Nucl. Phys. B 425, 634 (1994). https:// doi.org/10.1016/0550-3213(94)90291-7

9. G.M. Shore, Nucl. Phys. B 460, 379 (1996). https://doi.org/10. 1016/0550-3213(95)00646-X

10. R.D. Daniels, G.M. Shore, Phys. Lett. B 367, 75 (1996). https:// doi.org/10.1016/0370-2693(95)01468-3

11. S. Mohanty, A.R. Prasanna, Nucl. Phys. B 526, 501 (1998). https:// doi.org/10.1016/S0550-3213(98)00275-2

12. H.T. Cho, Phys. Rev. D 56, 6416 (1997). https://doi.org/10.1103/ PhysRevD.56.6416

13. R.G. Cai, Nucl. Phys. B 524, 639 (1998). https://doi.org/10.1016/ S0550-3213(98)00274-0

14. G.M. Shore, Nucl. Phys. B 605, 455 (2001). https://doi.org/10. 1016/S0550-3213(01)00137-7

15. G.M. Shore, Nucl. Phys. B 633, 271 (2002). https://doi.org/10. 1016/S0550-3213(02)00240-7

16. M.S. Turner, L.M. Widrow, Phys. Rev. D 37, 2743 (1988). https:// doi.org/10.1103/PhysRevD.37.2743

17. F.D. Mazzitelli, F.M. Spedalieri, Phys. Rev. D 52, 6694 (1995). https://doi.org/10.1103/PhysRevD.52.6694

18. G. Lambiase, A.R. Prasanna, Phys. Rev. D 70(6), 063502 (2004). https://doi.org/10.1103/PhysRevD.70.063502

19. A. Raya, J.E.M. Aguilar, M. Bellini, Phys. Lett. B 638, 314 (2006). https://doi.org/10.1016/j.physletb.2006.05.068

20. L. Campanelli, P. Cea, G.L. Fogli, L. Tedesco, Phys. Rev. D 77(12), 123002 (2008). https://doi.org/10.1103/PhysRevD.77.123002

21. K. Bamba, S.D. Odintsov, J. Cosmol. Astropart. Phys. 4, 024 (2008). https://doi.org/10.1088/1475-7516/2008/04/024

22. R. Lafrance, R.C. Myers, Phys. Rev. D 51, 2584 (1995). https:// doi.org/10.1103/PhysRevD.51.2584

23. M. Novello, L.A.R. Oliveira, J.M. Salim, Class. Quantum Gravity 13, 1089 (1996). https://doi.org/10.1088/0264-9381/13/5/022

24. F.W. Hehl, Y.N. Obukhov, in Gyros, Clocks, Interferometers: Testing Relativistic Gravity in Space, Lect. Notes in Phys., vol. 562, ed. by C. Lämmerzahl, C.W.F. Everitt, F.W. Hehl (Springer Verlag, Berlin, 2001), Lect. Notes in Phys., vol. 562, p. 479

25. Y. Itin, F.W. Hehl, Phys. Rev. D 68(12), 127701 (2003). https://doi. org/10.1103/PhysRevD.68.127701

26. S.K. Solanki, O. Preuss, M.P. Haugan, A. Gandorfer, H.P. Povel, P. Steiner, K. Stucki, P.N. Bernasconi, D. Soltau, Phys. Rev. D 69(6), 062001 (2004). https://doi.org/10.1103/PhysRevD.69.062001

27. O. Preuss, M.P. Haugan, S.K. Solanki, S. Jordan, Phys. Rev. D 70(6), 067101 (2004). https://doi.org/10.1103/PhysRevD.70. 067101

28. A.B. Balakin, J.P.S. Lemos, Class. Quantum Gravity 22, 1867 (2005). https://doi.org/10.1088/0264-9381/22/9/024

29. A.B. Balakin, V.V. Bochkarev, J.P.S. Lemos, Phys. Rev. D 77(8), 084013 (2008). https://doi.org/10.1103/PhysRevD.77.084013

30. T. Dereli, Ö. Sert, Eur. Phys. J. C 71, 1589 (2011). https://doi.org/ 10.1140/epjc/s10052-011-1589-2

31. A. Ritz, J. Ward, Phys. Rev. D 79(6), 066003 (2009). https://doi. org/10.1103/PhysRevD.79.066003

32. D.Z. Ma, Y. Cao, J.P. Wu, Phys. Lett. B 704, 604 (2011). https:// doi.org/10.1016/j.physletb.2011.09.058

33. J.P. Wu, Y. Cao, X.M. Kuang, W.J. Li, Phys. Lett. B 697, 153 (2011). https://doi.org/10.1016/j.physletb.2011.01.045

34. D. Momeni, M.R. Setare, Mod. Phys. Lett. A 26, 2889 (2011). https://doi.org/10.1142/S0217732311037169

35. D. Momeni, M.R. Setare, R. Myrzakulov, Int. J. Mod. Phys. A 27, 1250128 (2012). https://doi.org/10.1142/S0217751X1250128X

36. D. Momeni, N. Majd, R. Myrzakulov, Europhys. Lett. 97, 61001 (2012). https://doi.org/10.1209/0295-5075/97/61001

37. D. Roychowdhury, Phys. Rev. D 86(10), 106009 (2012). https:// doi.org/10.1103/PhysRevD.86.106009
38. Z. Zhao, Q. Pan, J. Jing, Phys. Lett. B 719, 440 (2013). https://doi. org/10.1016/j.physletb.2013.01.030

39. J. Jing, S. Chen, Q. Pan, Ann. Phys. 367, 219 (2016). https://doi. org/10.1016/j.aop.2016.01.015

40. S. Chen, J. Jing, Phys. Rev. D 88(6), 064058 (2013). https://doi. org/10.1103/PhysRevD.88.064058

41. S. Chen, J. Jing, Phys. Rev. D 90(12), 124059 (2014). https://doi. org/10.1103/PhysRevD.90.124059

42. S. Chen, J. Jing, Phys. Rev. D 89(10), 104014 (2014). https://doi. org/10.1103/PhysRevD.89.104014

43. H. Liao, S. Chen, J. Jing, Phys. Lett. B 728, 457 (2014). https:// doi.org/10.1016/j.physletb.2013.12.018

44. C. Darwin, Proc. R. Soc. Lond. Ser. A 249, 180 (1959). https://doi. org/10.1098/rspa.1959.0015

45. V. Bozza, Gen. Relativ. Gravity 42, 2269 (2010). https://doi.org/ 10.1007/s10714-010-0988-2

46. S. Chen, J. Jing, J. Cosmol. Astropart. Phys. 10, 002 (2015). https:// doi.org/10.1088/1475-7516/2015/10/002

47. X. Lu, F.W. Yang, Y. Xie, Eur. Phys. J. C 76, 357 (2016). https:// doi.org/10.1140/epjc/s10052-016-4218-2

48. S. Chen, S. Wang, Y. Huang, J. Jing, S. Wang, Phys. Rev. D 95(10), 104017 (2017). https://doi.org/10.1103/PhysRevD.95.104017

49. K.S. Virbhadra, D. Narasimha, S.M. Chitre, Astron. Astrophys. 337, 1 (1998)

50. K.S. Virbhadra, G.F.R. Ellis, Phys. Rev. D 62(8), 084003 (2000). https://doi.org/10.1103/PhysRevD.62.084003

51. A.O. Petters, Mon. Not. R. Astron. Soc. 338, 457 (2003). https:// doi.org/10.1046/j.1365-8711.2003.06065.x

52. C.R. Keeton, A.O. Petters, Phys. Rev. D 72(10), 104006 (2005). https://doi.org/10.1103/PhysRevD.72.104006

53. C.R. Keeton, A.O. Petters, Phys. Rev. D 73(4), 044024 (2006). https://doi.org/10.1103/PhysRevD.73.044024

54. C.R. Keeton, A.O. Petters, Phys. Rev. D 73(10), 104032 (2006). https://doi.org/10.1103/PhysRevD.73.104032

55. X.M. Deng, Y. Xie, Phys. Rev. D 93(4), 044013 (2016). https:// doi.org/10.1103/PhysRevD.93.044013

56. S. Weinberg, Gravitation and Cosmology: Principles and Applications of the General Theory of Relativity (Wiley, New York, 1972)

57. G. Li, X.M. Deng, Ann. Phys. 382, 136 (2017). https://doi.org/10. 1016/j.aop.2017.05.001

58. S. Refsdal, Mon. Not. R. Astron. Soc. 128, 295 (1964). https://doi. org/10.1093/mnras/128.4.295

59. S. Gillessen, P.M. Plewa, F. Eisenhauer, R. Sari, I. Waisberg, M. Habibi, O. Pfuhl, E. George, J. Dexter, S. von Fellenberg, T. Ott, R. Genzel, Astrophys. J. 837, 30 (2017). https://doi.org/10.3847/ $1538-4357 / \mathrm{aa} 5 \mathrm{c} 41$

60. GRAVITY Collaboration, Astron. Astrophys. 602, A94 (2017). https://doi.org/10.1051/0004-6361/201730838

61. W.J. Borucki, D. Koch, G. Basri, N. Batalha, T. Brown, D. Caldwell, J. Caldwell, J. Christensen-Dalsgaard, W.D. Cochran, E. DeVore, E.W. Dunham, A.K. Dupree, T.N. Gautier, J.C. Geary, R. Gilliland, A. Gould, S.B. Howell, J.M. Jenkins, Y. Kondo, D.W. Latham, G.W. Marcy, S. Meibom, H. Kjeldsen, J.J. Lissauer, D.G. Monet, D. Morrison, D. Sasselov, J. Tarter, A. Boss, D. Brownlee, T. Owen, D. Buzasi, D. Charbonneau, L. Doyle, J. Fortney, E.B. Ford, M.J. Holman, S. Seager, J.H. Steffen, W.F. Welsh, J. Rowe, H. Anderson, L. Buchhave, D. Ciardi, L. Walkowicz, W. Sherry, E. Horch, H. Isaacson, M.E. Everett, D. Fischer, G. Torres, J.A. Johnson, M. Endl, P. MacQueen, S.T. Bryson, J. Dotson, M. Haas, J. Kolodziejczak, J. van Cleve, H. Chandrasekaran, J.D. Twicken, E.V. Quintana, B.D. Clarke, C. Allen, J. Li, H. Wu, P. Tenenbaum, E. Verner, F. Bruhweiler, J. Barnes, A. Prsa, Science 327, 977 (2010). https://doi.org/ $10.1126 /$ science. 1185402

62. C. Alcock, C.W. Akerlof, R.A. Allsman, T.S. Axelrod, D.P. Bennett, S. Chan, K.H. Cook, K.C. Freeman, K. Griest, S.L. Marshall, H.S. Park, S. Perlmutter, B.A. Peterson, M.R. Pratt, P.J. 
Quinn, A.W. Rodgers, C.W. Stubbs, W. Sutherland, Nature 365, 621 (1993). https://doi.org/10.1038/365621a0

63. A. Udalski, M. Szymanski, J. Kaluzny, M. Kubiak, W. Krzeminski, M. Mateo, G.W. Preston, B. Paczynski, Acta Astron. 43, 289 (1993)

64. E. Aubourg, P. Bareyre, S. Bréhin, M. Gros, M. Lachièze-Rey, B. Laurent, E. Lesquoy, C. Magneville, A. Milsztajn, L. Moscoso, F. Queinnec, J. Rich, M. Spiro, L. Vigroux, S. Zylberajch, R. Ansari, F. Cavalier, M. Moniez, J.P. Beaulieu, R. Ferlet, P. Grison, A. VidalMadjar, J. Guibert, O. Moreau, F. Tajahmady, E. Maurice, L. Prévôt, C. Gry, Nature 365, 623 (1993). https://doi.org/10.1038/365623a0

65. C. Alard, J. Guibert, O. Bienayme, D. Valls-Gabaud, A.C. Robin, A. Terzan, E. Bertin, The Messenger 80, 31 (1995)

66. I.A. Bond, F. Abe, R.J. Dodd, J.B. Hearnshaw, M. Honda, J. Jugaku, P.M. Kilmartin, A. Marles, K. Masuda, Y. Matsubara, Y. Muraki, T. Nakamura, G. Nankivell, S. Noda, C. Noguchi, K. Ohnishi, N.J. Rattenbury, M. Reid, T. Saito, H. Sato, M. Sekiguchi, J. Skuljan, D.J. Sullivan, T. Sumi, M. Takeuti, Y. Watase, S. Wilkinson, R. Yamada, T. Yanagisawa, P.C.M. Yock, Mon. Not. R. Astron. Soc. 327, 868 (2001). https://doi.org/10.1046/j.1365-8711.2001. 04776.x
67. M.G. Navarro, D. Minniti, R. Contreras Ramos, Astrophys. J. Lett. 851, L13 (2017). https://doi.org/10.3847/2041-8213/aa9b29

68. M.A. Walker, Astrophys. J. 453, 37 (1995). https://doi.org/10. $1086 / 176367$

69. M. Dominik, K.C. Sahu, Astrophys. J. 534, 213 (2000). https://doi. org/10.1086/308716

70. S. Proft, M. Demleitner, J. Wambsganss, Astron. Astrophys. 536, A50 (2011). https://doi.org/10.1051/0004-6361/201117663

71. K.C. Sahu, H.E. Bond, J. Anderson, M. Dominik, Astrophys. J. 782, 89 (2014). https://doi.org/10.1088/0004-637X/782/2/89

72. N. Kains, A. Calamida, K.C. Sahu, S. Casertano, J. Anderson, A. Udalski, M. Zoccali, H. Bond, M. Albrow, I. Bond, T. Brown, M. Dominik, C. Fryer, M. Livio, S. Mao, M. Rejkuba, Astrophys. J. 843, 145 (2017). https://doi.org/10.3847/1538-4357/aa78eb

73. K.C. Sahu, J. Anderson, S. Casertano, H.E. Bond, P. Bergeron, E.P. Nelan, L. Pueyo, T.M. Brown, A. Bellini, Z.G. Levay, J. Sokol, M. Dominik, A. Calamida, N. Kains, M. Livio, Science 356, 1046 (2017). https://doi.org/10.1126/science.aal2879

74. M. Sereno, F. de Luca, Phys. Rev. D 74(12), 123009 (2006). https:// doi.org/10.1103/PhysRevD.74.123009 